\title{
Local $a b$ initio methods for calculating optical band gaps in periodic systems. I. Periodic density fitted local configuration interaction singles method for polymers
}

\author{
Marco Lorenz, Denis Usvyat, ${ }^{\text {a) }}$ and Martin Schütz \\ Institute for Physical and Theoretical Chemistry, Universität Regensburg, Universitätsstrasse 31, D-93040 \\ Regensburg, Germany
}

(Received 16 November 2010; accepted 21 January 2011; published online 1 March 2011)

\begin{abstract}
We present a density fitted local configuration interaction singles (CIS) method for calculating optical band gaps in 1D-periodic systems. The method is based on the Davidson diagonalization procedure, carried out in the reciprocal space. The one-electron part of the matrix-vector products is also evaluated in the reciprocal space, where the diagonality of the Fock matrix can be exploited. The contraction of the CIS vectors with the two electron integrals is performed in the direct space in the basis of localized occupied (Wannier) and virtual (projected atomic) orbitals. The direct space approach allows to utilize the sparsity of the integrals due to the local representation and locality of the exciton. The density fitting approximation employed for the two electron integrals reduces the nominal scaling with unit cell size to $\mathcal{O}\left(N^{4}\right)$. Test calculations on a series of prototypical systems demonstrate that the method in its present stage can be used to calculate the excitonic band gaps of polymers with up to a few dozens of atoms in the cell. The computational cost depends on the locality of the exciton, but even relatively delocalized excitons occurring in the polybiphenyl in the parallel orientation, can be routinely treated with this method. () 2011 American Institute of Physics. [doi:10.1063/1.3554209]
\end{abstract}

\section{INTRODUCTION}

Quantum chemistry offers a wide range of ab initio techniques of increasing accuracy and cost for calculating the electronic structure of both ground and excited states of molecules. The lowest ranking in the hierarchy occupy the relatively inexpensive and at the same time not very accurate Hartree-Fock (HF) (for the ground state) and configuration interaction singles (CIS) (for the excited states) methods. Among the highly accurate, but at the same time still feasible, at least for not very extended systems, methods one can mention the $\operatorname{CCSD}(\mathrm{T})$ and MRCI- "golden standards" for the single-reference ground state and multireference ground and excited state calculations, respectively. As a reasonable compromise between accuracy and cost of the calculations one usually considers second order methods like MP2 for the ground state and CC2 response, ${ }^{1} \mathrm{ADC}(2),{ }^{2} \mathrm{CIS}(\mathrm{D})^{3}$ or $\mathrm{CASPT}^{4}$ for the excited states, which can be applied to rather extended systems, especially when combined with local schemes. ${ }^{5,6}$

For periodic systems, quantum chemical treatments are much more complicated and costly. As a consequence, the density functional theory (DFT) is the most common technique used for ground state periodic calculations. Recently, the MP2 method has been generalized to the $3 \mathrm{D}$ periodic case within the Laplace-transform, ${ }^{7}$ local, ${ }^{8}$ and canonical plane wave $^{9}$ formulations. Nevertheless, so far most of the quantum chemical approaches to the ground state of periodic systems rely on finite cluster models. ${ }^{10-12}$ For excited states,

\footnotetext{
${ }^{a)}$ Electronic mail: denis.usvyat@chemie.uni-regensburg.de.
}

the finite cluster approach ${ }^{13}$ is more problematic, making periodic schemes preferable. Most of these methods use the Green's function formalism, ${ }^{14-20}$ including MP2-based quasiorbital approaches. ${ }^{7,21-24}$ Periodic CIS and time dependent HF (TD-HF) methods and their DFT counterparts (TDA, TDDFT) have been formulated ${ }^{25}$ and implemented for 1D periodic systems. ${ }^{21,25-29}$ The periodic CIS treatment has been complemented with schemes, allowing for a low level inclusion of correlation effects. ${ }^{21,22}$ The most elaborate scheme within the theoretical description of excited states in periodic systems presently is EOM-CC theory with periodic boundary conditions, which was applied to polyethylene. ${ }^{30}$ This method turned out to be computationally extremely costly, but at the same time provided accurate results. As an example for an early calculation of excitonic effects in a 3D solid one can mention a TD-HF method employing approximate Wannier functions of the valence and lowest conduction bands, which has been applied to diamond. ${ }^{31}$ Nowadays, the excitonic states are usually studied by solving the Bethe-Salpeter equation, for quasi-particles obtained within DFT/GW approach. ${ }^{16,17,32-36}$ This method is quite expensive, but delivers accurate excitation energies. Formally, it reduces to a TD-HF- or CIS-like equation but with a screened rather than the bare Coulomb kernel.

Despite the existence of a number of periodic excited state methods the Kohn-Sham (more rarely HF) HOMOLUMO difference still remains the most popular estimate for the excitation energies in periodic systems. The TD-DFT method, which has proven to be quite successful in molecular studies is not as useful in the periodic case. In fact, the intrinsic problem of the standard DFT - the self interaction error, 
which causes a substantial underestimation of charge transfer state energies by TD-DFT, can lead to extreme consequences in infinite systems. As was shown in Refs. 26 and 37, the lowest excitation energy of semilocal TD-DFT in a crystal is equal to the ordinary DFT HOMO-LUMO difference. ${ }^{37}$ This fact questions both the usefulness and adequacy of the TD-DFT approach in the periodic context. Indeed, since the TD-DFT result can be obtained with much smaller effort by performing a simple DFT calculation, it, at least for the lowest excited state, implicitly ignores the electron-hole attraction, essential for the correct description of an exciton.

This work is the first step toward the development of a hierarchy of quantum chemical excited state methods for periodic systems. Such methods would be a valuable complement to the existing Green's function based approaches (vide supra). In this paper we describe a local CIS method applicable to polymers. This method is the lowest in the hierarchy and is not expected to provide high accuracy, since electron correlation effects are not included. At the same time, it captures the excitonic nature of the excited state and can be a good starting point for a more elaborate and accurate correlated treatment, which is planned to be implemented at a later stage.

Our formalism is based on Gaussian-type-orbital (GTO) basis sets and localized orbitals both for occupied and virtual states. The local representation allows for significant computational savings compared to the canonical one for strongly and intermediately bound excitons and fits into the intuitive picture of a localized hole interacting with a localized electron. The attractive feature of the local description of excitons has been noticed very early, so that Wannier functions (WFs)-localized orbitals in periodic systems-were introduced for the first time in the conjunction with this particular problem. ${ }^{38}$ However this approach remained illustrative, rather than practical due to the difficulty in obtaining well localized virtual functions. In the current work we apply a technique, adopted from the local correlation methods for the ground state ${ }^{39,40}$ where the virtual space is spanned by mutually nonorthogonal, and even partially redundant, but well localized projected atomic orbitals (PAOs).

The paper is organized as follows. In Sec. II we present the general formalism of the periodic local CIS method. The details of the implementation are given in Sec. III. The results of test calculations and their analysis are reported in Sec. IV. Section V concludes the paper.

Throughout the paper we use the following convention: the indices $(i, j, \ldots)$ and $(a, b, \ldots)$ denote Wannier functions and projected atomic orbitals, respectively, or their Fourier images. The indices $(\bar{i}, \bar{j}, \ldots)$ and $(\bar{a}, \bar{b}, \ldots)$ label the canonical occupied and virtual orbitals. $(P, Q, \ldots)$ represent auxiliary fitting functions and $(\mu, v, \ldots)$ atomic orbitals (AOs). The calligraphic indices $(\mathcal{I}, \mathcal{J}, \ldots),(\mathcal{A}, \mathcal{B}, \ldots),(\mathcal{P}, \mathcal{Q}, \ldots)$ and $(\mathcal{M}, \mathcal{N}, \ldots)$ identify the lattice vectors to the cells, where the functions $(i, j, \ldots),(a, b \ldots),(P, Q, \ldots)$ and $(\mu, v, \ldots)$ are centered. The vectors $\left(\mathbf{k}_{i}, \mathbf{k}_{j}, \ldots\right),\left(\mathbf{k}_{a}, \mathbf{k}_{b}, \ldots\right)$ and $\left(\mathbf{k}_{P}, \mathbf{k}_{Q}, \ldots\right)$ indicate the wave-vectors from the first Brillouin zone corresponding to the occupied, virtual, or auxiliary functions (to be referred to in the following as k-vectors or k-points). With the term $\mathbf{k}$-mesh or $\mathbf{k}$-net we will designate the actual sets of the k-points used in the calculations. The chemical (Mulliken) notation is employed for the two-electron integrals.

\section{PERIODIC LOCAL CIS METHOD}

\section{A. The excitonic $\mathrm{CIS}$ wavefunction}

The CIS wavefunction $\left|\Psi_{\text {exc }}\left(\mathbf{k}_{\text {exc }}\right)\right\rangle$ for an exciton with the translational symmetry $\mathbf{k}_{\mathrm{exc}}$ is defined as

$$
\left|\Psi_{\text {exc }}\left(\mathbf{k}_{\text {exc }}\right)\right\rangle=\hat{C}_{1}\left(\mathbf{k}_{\text {exc }}\right)|0\rangle,
$$

where $|0\rangle$ is the Hartree-Fock determinant and $\hat{C}_{1}\left(\mathbf{k}_{\text {exc }}\right)$ is a linear combination of single excitation operators. It can be considered in the local basis, defined in the direct space, as

$$
\hat{C}_{1}\left(\mathbf{k}_{\mathrm{exc}}\right)=\sum_{i \mathcal{I} a \mathcal{A}} c_{a \mathcal{A}}^{i \mathcal{I}}\left(\mathbf{k}_{\mathrm{exc}}\right) \hat{\tau}_{i \mathcal{I}}^{a \mathcal{A}},
$$

where $\hat{\tau}_{i \mathcal{I}}^{a \mathcal{A}}$ is an excitation operator in second quantization, which excites an electron from the local occupied orbital (Wannier function) $i$ located in the $\mathcal{I}$-cell into a projected atomic orbital $a$ located in the $\mathcal{A}$-cell, and $c_{a \mathcal{A}}^{i \mathcal{I}}\left(\mathbf{k}_{\mathrm{exc}}\right)$ are the corresponding amplitudes.

The wavefunction can alternatively and equivalently be written in the canonical basis:

$$
\hat{C}_{1}\left(\mathbf{k}_{\mathrm{exc}}\right)=\sum_{\bar{i} \mathbf{k}_{i} \bar{a} \mathbf{k}_{a}} c_{\bar{a} \mathbf{k}_{a}}^{\bar{i} \mathbf{k}_{i}}\left(\mathbf{k}_{\mathrm{exc}}\right) \hat{\tau}_{\bar{i} \mathbf{k}_{i}}^{\bar{a} \mathbf{k}_{a}},
$$

where $\hat{\tau}_{\bar{i} \mathbf{k}_{i}}^{\bar{a} \mathbf{k}_{a}}$, which excites an electron from the occupied Bloch orbital $\bar{i}$ with translational symmetry $\mathbf{k}_{i}$ into the virtual Bloch orbital $\bar{a}$ with translational symmetry $\mathbf{k}_{a}$, is weighted with the corresponding amplitude $c_{\bar{a} \mathbf{k}_{a}}^{\bar{i} \mathbf{k}_{i}}\left(\mathbf{k}_{\text {exc }}\right)$.

The Bloch theorem states that for a $\Gamma$-point exciton, i.e., $\mathbf{k}_{\mathrm{exc}}=0$, a Bravais lattice translation $\hat{T}_{\mathbf{R}}$ does not affect the wavefunction:

$$
\hat{T}_{\mathbf{R}}\left|\Psi_{\text {exc }}\left(\mathbf{k}_{\text {exc }}=0\right)\right\rangle=\hat{T}_{\mathbf{R}}\left|\Psi_{\text {exc }}^{\Gamma}\right\rangle=\left|\Psi_{\text {exc }}^{\Gamma}\right\rangle .
$$

In the following we focus on the excitons of this particular symmetry and therefore omit for brevity the index $\mathbf{k}_{\text {exc }}$.

From Eqs. (2) and (4) it follows that the CIS amplitudes in the local representation possess the translational invariance:

$$
c_{a \mathcal{A}}^{i \mathcal{I}}=c_{a(\mathcal{A} \ominus \mathcal{I})}^{i} .
$$

This means that only the amplitudes, where, e.g., the occupied index is restricted to the reference cell are symmetry unique and therefore need to be calculated.

The CIS amplitudes for the $\Gamma$-point excitons in canonical representation (and generally in the $\mathbf{k}$-space representation), also have a symmetry restriction:

$$
c_{\bar{a} \mathbf{k}_{a}}^{\bar{i} \mathbf{k}_{i}}= \begin{cases}c_{\bar{a}}^{\bar{i}}(\mathbf{k}) & \text { if } \mathbf{k}_{i}=\mathbf{k}_{a}=\mathbf{k}, \\ 0 & \text { otherwise. }\end{cases}
$$

Thus, only the coefficients corresponding to vertical (in kspace sense) excitations are nonzero and just one Fourier transform between direct and reciprocal space has to be carried out for the amplitudes (vide infra). The corresponding excitation operator from Eqs. (2) and (3) then reduces to

$$
\hat{C}_{1}=\sum_{i a \mathcal{A}} c_{a \mathcal{A}}^{i} \sum_{\mathcal{I}} \hat{\tau}_{i \mathcal{I}}^{a(\mathcal{A}+\mathcal{I})}=\sum_{\bar{i} \bar{a} \mathbf{k}} c_{\bar{a}}^{\bar{i}}(\mathbf{k}) \hat{\tau}_{\bar{i}}^{\bar{a}}(\mathbf{k}) .
$$




\section{B. The CIS equations}

Projecting the excitonic CIS Schrödinger equation (with a normal ordered Hamiltonian $\hat{H}_{N}$ ),

$$
\hat{H}_{N} \hat{C}_{1}|0\rangle=\omega_{\mathrm{CIS}} \hat{C}_{1}|0\rangle,
$$

onto singly excited determinants $\left\langle\Phi_{i}^{a \mathcal{A}}\right|$ or $\left\langle\Phi_{\bar{i}}^{\bar{a}}(\mathbf{k})\right|$ yields

$$
\begin{aligned}
& \sum_{b \mathcal{B}} F_{a \mathcal{A} b \mathcal{B}} c_{b \mathcal{B}}^{i}-\sum_{j \mathcal{J} b \mathcal{B}} S_{a \mathcal{A} b \mathcal{B}}^{\mathrm{PAO}} c_{b \mathcal{B}}^{j \mathcal{J}} F_{j \mathcal{J} i} \\
& +2 \sum_{j \mathcal{J} b \mathcal{B}}(a \mathcal{A} i \mid j \mathcal{J} b \mathcal{B}) c_{b \mathcal{B}}^{j \mathcal{J}}-\sum_{j \mathcal{J} b \mathcal{B}}(j \mathcal{J} i \mid a \mathcal{A} b \mathcal{B}) c_{b \mathcal{B}}^{j \mathcal{J}} \\
& =\omega_{\mathrm{CIS}} c_{a \mathcal{A}}^{i}
\end{aligned}
$$

or

$$
\begin{aligned}
& \left(\varepsilon_{\bar{a}}(\mathbf{k})-\varepsilon_{\bar{i}}(\mathbf{k})\right) c_{\bar{a}}^{\bar{i}}(\mathbf{k}) \\
& +2 \sum_{\bar{j} \bar{b} \mathbf{k}^{\prime}}\left(\bar{a} \mathbf{k} \bar{i} \mathbf{k} \mid \bar{j} \mathbf{k}^{\prime} \bar{b} \mathbf{k}^{\prime}\right) c_{\bar{b}}^{\bar{j}}\left(\mathbf{k}^{\prime}\right)-\sum_{\bar{j} \bar{b} \mathbf{k}^{\prime}}\left(\bar{j} \mathbf{k}^{\prime} \bar{i} \mathbf{k} \mid \bar{a} \mathbf{k} \bar{b} \mathbf{k}^{\prime}\right) c_{\bar{b}}^{\bar{j}}\left(\mathbf{k}^{\prime}\right) \\
& \quad=\omega_{\mathrm{CIS}} c_{\bar{a}}^{\bar{i}}(\mathbf{k})
\end{aligned}
$$

respectively. Here the Fock matrix is denoted by $\mathbf{F}$ and the orbital energies by $\varepsilon$. Since in the present formalism the virtual local orbitals are not orthogonal (vide infra) the corresponding overlap matrix $\mathbf{S}^{\mathrm{PAO}}$ appears. For the triplet state the first two-electron integral on the left hand side of Eqs. (9) and (10) vanishes because of the orthogonality between the spin states.

On the one hand, the contraction with the Fock matrix becomes trivial in the canonical representation [Eq. (10)]. On the other hand, the evaluation of the two-electron part in the direct space and local representation, i.e., according to Eq. (9), is preferable. Indeed, the locality of the orbitals allows for exploitation of the sparsity in the integrals and the CIS coefficients. Moreover, there is no need for a slowly convergent Fourier transform of the AO twoelectron integrals into the reciprocal space as in Ref. 26. And finally, efficient density fitting techniques for the twoelectron integrals in the local representation, as employed in the periodic local MP2 method ${ }^{8,41-43}$ can be applied to the CIS formalism (vide infra).

Separating thus the Fock matrix and two-electron parts and introducing the matrix notation one can write Eqs. (9) and (10) as

$$
\mathbf{H c}=\mathbf{F} \mathbf{c}+\mathbf{V} \mathbf{c}=\omega_{\mathrm{CIS}} \mathbf{c} .
$$

\section{Davidson procedure}

Equation (11) is the standard CI Hermitian eigenvalue problem, with the lowest eigenvalue corresponding to the optical band gap at the $\Gamma$-point. In order to calculate it we employ the Davidson diagonalization method. ${ }^{44}$ This method represents a combination of an iterative updating of the eigenvector and a matrix diagonalization in the small subspace spanned by the approximate eigenvectors generated by the iterative procedure. Its convergence is much superior to a straight iterative method. A similar procedure was used by Roos and Siegbahn in their direct CI method. ${ }^{45}$ The smaller space Hamilton matrix (to be referred to as "small h-matrix") in the $n$th iteration can be written as

$$
h_{r s}^{(n)}=\Delta \mathbf{c}^{\dagger(r)}(\mathbf{H} \Delta \mathbf{c})^{(s)},
$$

where the CIS trial vectors are denoted by $\Delta \mathbf{c}$, and the upperscript indices count the iterations $(r, s=1,2, \ldots, n-1)$.

Exploiting the diagonality of the Fock matrix in the canonical basis, we evaluate the update or the new trial vector for the next iteration within first order perturbation theory in the Møller-Plesset partitioning as

$$
\left(\Delta \mathbf{c}^{(n)}\right)_{\bar{a}}^{\bar{i}}(\mathbf{k})=-\frac{\left(\mathbf{H c}^{(n)}\right)_{\bar{i}}^{\bar{a}}(\mathbf{k})-\omega_{\mathrm{CIS}}^{(n)}\left(\mathbf{c}^{(n)}\right)_{\bar{a}}^{\bar{i}}(\mathbf{k})}{\varepsilon_{\bar{a}}(\mathbf{k})-\varepsilon_{\bar{i}}(\mathbf{k})-\omega_{\mathrm{CIS}}^{(n)}} .
$$

Here, $\mathbf{c}^{(n)}$ denotes the vector in the full canonical space constructed from the eigenvector of the small $\mathbf{h}$-matrix, corresponding to its lowest eigenvalue $\omega_{\text {CIS }}^{(n)}$. The new trial vector $\Delta \mathbf{c}^{(n)}$ is orthogonalized to the trial vectors of the previous iterations and normalized.

Once the trial vector for the $n$th iteration has been obtained, the Fock part $\mathbf{F} \Delta \mathbf{c}^{(n)}$ is directly calculated. Transformation of the trial vector to the direct space allows to evaluate the two-electron part $\mathbf{V} \Delta \mathbf{c}^{(n)}$, which then is subsequently transformed back to the canonical representation and added to the Fock part. Finally, the small h-matrix for the next iteration is constructed via (12) in the canonical basis. The particular choice of the local basis and the corresponding transformations, essential for the scheme are discussed in Sec. ID.

\section{Local representation}

\section{Definition of the local orbitals}

As mentioned above the introduction of the local basis and the contraction of the CIS coefficients with the two electron integrals in the direct space is one of the key features of the present scheme. Although formally straightforward, this scheme can be efficient in practice only when both the occupied and virtual orbitals are well-localized, which is not trivial to achieve, especially for the virtual orbitals.

Construction of well-localized occupied orbitals in periodic systems - Wannier functions - is a more complicated matter than in molecules. However, in the last two decades several efficient methods for their generation have been proposed. ${ }^{46,47}$ For the virtual states, the same localization schemes can formally be applied, but the spread of such orbitals remains relatively large. The reasons for the poor localization are the mutual orthogonality constraint entering the formalism of most of the localization methods together with the greater number of virtual states compared to the occupied ones, and, to some extent, an intrinsic more delocalized character of the former. In the present work we use mutually nonorthogonal PAOs, which have shown to be instrumental in local correlation methods for the ground state. ${ }^{8,40}$ In this case the virtual manifold is spanned by AOs projected onto the virtual space. The orbitals constructed in such a way are usually quite well localized, but at the same time nonorthogonal and moreover redundant. The latter property introduces some complications in the formalism, however, this is offset by the gain due to localization.

Wannier functions $\left|\phi_{i \mathcal{I}}^{\mathrm{WF}}\right\rangle$ can be written as linear combinations of canonical Bloch functions $\left|\phi_{\bar{i}}^{\mathrm{CAN}}(\mathbf{k})\right\rangle$ : 


$$
\left|\phi_{i \mathcal{I}}^{\mathrm{WF}}\right\rangle=\frac{1}{\sqrt{n_{\mathbf{k}}}} \sum_{\mathbf{k}} \exp \left(-\imath \mathbf{k} \mathbf{R}_{\mathcal{I}}\right) \sum_{\bar{i}} W_{\bar{i} i}(\mathbf{k})\left|\phi_{\bar{i}}^{\mathrm{CAN}}(\mathbf{k})\right\rangle .
$$

Here, $n_{\mathbf{k}}$ is the number of points in the $\mathbf{k}$-mesh chosen for the discrete Fourier transform. The WFs are localized using the transformation matrices $W(\mathbf{k})$, which mix the states of the occupied bands. As in the CRYSCOR's periodic local MP2 approach, the Wannier functions are generated using the CRYSTAL $\operatorname{code}^{48}$ (vide infra). Mutual orthogonality of the WFs implies the unitarity of the matrices $\mathbf{W}(\mathbf{k})$ :

$$
\mathbf{W}^{-1}(\mathbf{k})=\mathbf{W}^{\dagger}(\mathbf{k}) \text {. }
$$

For convenience we introduce now the so called quasiBloch functions

$$
\left|\phi_{i}^{\mathrm{WF}}(\mathbf{k})\right\rangle=\sum_{\bar{i}} W_{\bar{i} i}(\mathbf{k})\left|\phi_{\bar{i}}^{\mathrm{CAN}}(\mathbf{k})\right\rangle,
$$

which due to the unitarity of the discrete Fourier transform are the Fourier images of the WFs

$$
\left|\phi_{i}^{\mathrm{WF}}(\mathbf{k})\right\rangle=\frac{1}{\sqrt{n_{\mathbf{k}}}} \sum_{\mathcal{I}} \exp \left(\imath \mathbf{k} \mathbf{R}_{\mathcal{I}}\right)\left|\phi_{i \mathcal{I}}^{\mathrm{WF}}\right\rangle .
$$

The unitarity of the $\mathbf{W}(\mathbf{k})$ matrices [Eq. (15)] allows to trivially invert equation (16),

$$
\left|\phi_{\bar{i}}^{\mathrm{CAN}}(\mathbf{k})\right\rangle=\sum_{i} W_{i \bar{i}}^{\dagger}(\mathbf{k})\left|\phi_{i}^{\mathrm{WF}}(\mathbf{k})\right\rangle .
$$

The PAOs are evaluated in a principally different way as compared to the WFs. Denoting the projector onto the HF occupied manifold as $\hat{P}$, and a $\mu$ th atomic orbital centered in a $\mathcal{M}$ th cell as $\phi_{\mu \mathcal{M}}^{\mathrm{AO}}$ we define the PAOs as ${ }^{39,40}$

$$
\left|\phi_{a \mathcal{A}}^{\mathrm{PAO}}\right\rangle=\left.(1-\hat{P})\left|\phi_{\mu \mathcal{M}}^{\mathrm{AO}}\right\rangle\right|_{a=\mu, \mathcal{A}=\mathcal{M}} .
$$

By construction they span the virtual space and are inherently local because of the locality of the AOs and of the projector $\hat{P} .{ }^{49}$ At the same time, due to the nonorthogonality of the AOs, the PAOs remain nonorthogonal as well. Moreover, it is clear from Eq. (19) that the number of PAOs is equal to the number of AOs and thus larger than the number of the virtual orbitals, which means that the PAOs form a redundant set. It is convenient to use un-normalized PAOs, otherwise the symmetry properties of the underlying AOs are lost.

Since the canonical virtual orbitals and the PAOs span the same space, the latter can be expressed via the former as in Eq. (14),

$\left|\phi_{a \mathcal{A}}^{\mathrm{PAO}}\right\rangle=\frac{1}{\sqrt{n_{\mathbf{k}}}} \sum_{\mathbf{k}} \exp \left(-\imath \mathbf{k} \mathbf{R}_{\mathcal{A}}\right) \sum_{\bar{a}} Q_{\bar{a} a}(\mathbf{k})\left|\phi_{\bar{a}}^{\mathrm{CAN}}(\mathbf{k})\right\rangle$,

and the Fourier images of the PAOs are obtained as

$$
\left|\phi_{a}^{\mathrm{PAO}}(\mathbf{k})\right\rangle=\sum_{\bar{a}} Q_{\bar{a} a}(\mathbf{k})\left|\phi_{\bar{a}}^{\mathrm{CAN}}(\mathbf{k})\right\rangle .
$$

The $\mathbf{Q}$ transformation is given by rectangular matrices $Q_{a \bar{a}}(\mathbf{k})$ with the property

$$
\mathbf{Q}^{\dagger}(\mathbf{k}) \mathbf{Q}(\mathbf{k})=\mathbf{S}^{\mathrm{PAO}}(\mathbf{k}),
$$

where $\mathbf{S}^{\mathrm{PAO}}(\mathbf{k})$ is the Fourier image of the PAO overlap matrix:

$$
S_{a b}^{\mathrm{PAO}}(\mathbf{k})=\left\langle\phi_{a}^{\mathrm{PAO}}(\mathbf{k}) \mid \phi_{b}^{\mathrm{PAO}}(\mathbf{k})\right\rangle
$$

$$
=\sum_{\mathcal{B}} \exp \left(\imath \mathbf{k} \mathbf{R}_{\mathcal{B}}\right)\left\langle\phi_{a 0}^{\mathrm{PAO}} \mid \phi_{b \mathcal{B}}^{\mathrm{PAO}}\right\rangle .
$$

The Fourier transform (20) is again easily inverted,

$$
\left|\phi_{a}^{\mathrm{PAO}}(\mathbf{k})\right\rangle=\frac{1}{\sqrt{n_{\mathbf{k}}}} \sum_{\mathcal{A}} \exp \left(\iota_{\mathbf{k}} \mathbf{R}_{\mathcal{A}}\right)\left|\phi_{a \mathcal{A}}^{\mathrm{PAO}}\right\rangle .
$$

Since the sets of PAOs or their Fourier images are redundant, the inverse of $\mathbf{S}^{\mathrm{PAO}}(\mathbf{k})$ does not exist (only the pseudoinverse!) and inversion of Eq. (21) becomes nonstraightforward. In order to carry it out we introduce the rectangular matrix

$$
\overline{\mathbf{Q}}(\mathbf{k})=\mathbf{X}(\mathbf{k}) \mathbf{Q}^{\dagger}(\mathbf{k}),
$$

( $\mathbf{X}$ is a yet unspecified matrix in the basis of the PAO reciprocal images) with the property

$$
\sum_{a \bar{a}}\left|\phi_{a}^{\mathrm{PAO}}(\mathbf{k})\right\rangle \bar{Q}_{a \bar{a}}(\mathbf{k}) Q_{\bar{a} c}(\mathbf{k})=\left|\phi_{c}^{\mathrm{PAO}}(\mathbf{k})\right\rangle .
$$

Multiplying Eq. (26) with the bra $\mathrm{PAO}\left\langle\phi_{d}^{\mathrm{PAO}}(\mathbf{k})\right|$ and integrating yields

$\mathbf{S}^{\mathrm{PAO}}(\mathbf{k}) \overline{\mathbf{Q}}(\mathbf{k}) \mathbf{Q}(\mathbf{k})=\mathbf{S}^{\mathrm{PAO}}(\mathbf{k}) \mathbf{X}(\mathbf{k}) \mathbf{S}^{\mathrm{PAO}}(\mathbf{k})=\mathbf{S}^{\mathrm{PAO}}(\mathbf{k})$,

defining the matrix $\mathbf{X}(\mathbf{k})$ as a pseudoinverse of the reciprocal image of the PAO overlap $\mathbf{S}^{\mathrm{PAO}}(\mathbf{k}){ }^{50}$ Expressing the right hand side of Eq. (26) via the canonical virtuals according to Eq. (21), one sees that the matrix $\overline{\mathbf{Q}}$ transforms the PAOs to the canonical basis,

$$
\left|\phi_{\bar{a}}^{\mathrm{CAN}}(\mathbf{k})\right\rangle=\sum_{a} \bar{Q}_{a \bar{a}}(\mathbf{k})\left|\phi_{a}^{\mathrm{PAO}}(\mathbf{k})\right\rangle .
$$

\section{Transformation of the amplitude vectors and matrix-vector products}

Now we are in a position to determine the form of the transformations for the CIS vectors $\mathbf{c}$ and $\Delta \mathbf{c}$ and the matrixvector products $\mathbf{V} \Delta \mathbf{c}$. According to the scheme described in Sec. II C, the trial vectors are transformed in each iteration from the canonical representation to the local one, while the matrix-vector products, evaluated in the direct space, are transformed back. If the starting trial vector is constructed in the direct space (vide infra) the local-canonical transformation has to be carried out for that vector also.

As is seen from Eqs. (9) or (10) the Vc vectors are transformed like ("ket") Wannier functions (the occupied index) and complex conjugated ("bra") PAOs (the virtual index). The direct space translational symmetry unique vectors have one of the indices, in our case the WF index, restricted to the reference cell (see Sec. II A). Therefore, the Fourier transform of the vectors involves only the sum over the PAO cell index or PAO k vector complex conjugated with respect to Eqs. (24) and (20):

$$
\begin{aligned}
& (\mathbf{V c})_{i}^{a}(\mathbf{k})=\frac{1}{\sqrt{n_{\mathbf{k}}}} \sum_{\mathcal{A}} \exp \left(-l \mathbf{k} \mathbf{R}_{\mathcal{A}}\right)(\mathbf{V} \mathbf{c})_{i}^{a \mathcal{A}}, \\
& (\mathbf{V c})_{i}^{a \mathcal{A}}=\frac{1}{\sqrt{n_{\mathbf{k}}}} \sum_{\mathbf{k}} \exp \left(\imath \mathbf{k} \mathbf{R}_{\mathcal{A}}\right)(\mathbf{V c})_{i}^{a}(\mathbf{k}) .
\end{aligned}
$$

Next, according to Eqs. (16), (18), (21) and (28) the transformations between the canonical and the local reciprocal-image 
representations are then given by

$$
\begin{aligned}
& (\mathbf{V c})_{\bar{i}}^{\bar{a}}(\mathbf{k})=\sum_{i a} \bar{Q}_{a \bar{a}}^{*}(\mathbf{k}) W_{i \bar{i}}^{\dagger}(\mathbf{k})(\mathbf{V} \mathbf{c})_{i}^{a}(\mathbf{k}), \\
& (\mathbf{V c})_{i}^{a}(\mathbf{k})=\sum_{\bar{i} \bar{a}} Q_{\bar{a} a}^{*}(\mathbf{k}) W_{\bar{i} i}(\mathbf{k})(\mathbf{V} \mathbf{c})_{\bar{i}}^{\bar{a}}(\mathbf{k}) .
\end{aligned}
$$

Having established these relations, we can focus on the transformation laws for the CIS vectors. According to Eq. (12), the Hermitian conjugate of the trial CIS vectors $\left(\Delta \mathbf{c}^{*}\right)_{a \mathcal{A}}^{i}$ or $\left(\mathbf{c}^{*}\right)_{a \mathcal{A}}^{i}$ transforms contravariantly as opposed to the covariant vector $(\mathbf{V c}) .{ }^{50}$ Therefore, the expressions for the transformations of $\left(\mathbf{c}^{*}\right)_{a}^{i}(\mathbf{k})$ and $\left(\mathbf{c}^{*}\right)_{\bar{a}}^{\bar{i}}(\mathbf{k})$ can be obtained directly from Eqs. (31) and (32), which after the complex conjugation of the left and right hand sides read

$$
\begin{aligned}
& (\mathbf{c})_{\bar{a}}^{\bar{i}}(\mathbf{k})=\sum_{i a} Q_{\bar{a} a}(\mathbf{k}) W_{i \bar{i}}^{\dagger}(\mathbf{k})(\mathbf{c})_{a}^{i}(\mathbf{k}), \\
& (\mathbf{c})_{a}^{i}(\mathbf{k})=\sum_{\bar{i} \bar{a}} \bar{Q}_{a \bar{a}}(\mathbf{k}) W_{\bar{i} i}(\mathbf{k})(\mathbf{c})_{\bar{a}}^{\bar{i}}(\mathbf{k}) .
\end{aligned}
$$

The same argument holds for the Fourier transformations of the CIS vectors.

$$
\begin{aligned}
& (\mathbf{c})_{a}^{i}(\mathbf{k})=\frac{1}{\sqrt{n_{\mathbf{k}}}} \sum_{\mathcal{A}} \exp \left(-l \mathbf{k} \mathbf{R}_{\mathcal{A}}\right)(\mathbf{c})_{a \mathcal{A}}^{i}, \\
& (\mathbf{c})_{a \mathcal{A}}^{i}=\frac{1}{\sqrt{n_{\mathbf{k}}}} \sum_{\mathbf{k}} \exp \left(l \mathbf{k} \mathbf{R}_{\mathcal{A}}\right)(\mathbf{c})_{a}^{i}(\mathbf{k}) .
\end{aligned}
$$

\section{E. Calculation of the Vc terms}

As was already discussed above, the two-electron integral part of the matrix-vector products is evaluated in the direct space. One of the main features of such a treatment is the possibility to use the periodic density fitting techniques as already devised for the periodic local MP2 method. ${ }^{8,41,43}$ Vc consists of two distinct contributions ( $\left.{ }^{\mathrm{coul}} \mathbf{V} \mathbf{c}\right)$ and $\left({ }^{\mathrm{exch}} \mathbf{V} \mathbf{c}\right)$ :

$$
\begin{aligned}
&\left({ }^{\text {coul }} \mathbf{V c}\right)_{i}^{a \mathcal{A}}=\sum_{j \mathcal{J} b \mathcal{B}}(a \mathcal{A} i \mid j \mathcal{J} b \mathcal{B}) c_{b \mathcal{B}}^{j \mathcal{J}}, \\
&\left({ }^{\operatorname{exch}} \mathbf{V c}\right)_{i}^{a \mathcal{A}}=\sum_{j \mathcal{J} b \mathcal{B}}(j \mathcal{J} i \mid a \mathcal{A} b \mathcal{B}) c_{b \mathcal{B}}^{j \mathcal{J}} .
\end{aligned}
$$

By virtue of the time reversal symmetry, the direct space quantities can be chosen real. The permutation of the "bra" and "ket" functions in the integrals is thus allowed.

The first term (37), appearing only in the singlet case, describes the Coulomb interaction between the neutral charge clouds of all the electron-hole pairs within the chosen basis. The corresponding diagram can be interpreted as hopping between electron-hole pairs driven by their mutual Coulomb (or, in the long range, dipole-dipole) interaction. The second term (38), which originates from the exchange-type diagram represents the Coulomb attraction between the hole and electron distributions. This is actually the key term in the noncorrelated description of an exciton, which is missing in time-dependent DFT, based on semilocal functionals. ${ }^{37}$
The computational cost of both contractions (37) and (38) scales nominally as $\mathcal{O}\left(N^{4}\right)$ with respect to the unit-cell size. However, the transformation of the four-index two-electron integrals to the WF-PAO basis scales as $\mathcal{O}\left(N^{5}\right)$, which constitutes the scaling bottleneck of the whole method. The density fitting approximation ${ }^{51}$ factorizes the four-index integrals through three-index quantities and reduces the overall scaling to $\mathcal{O}\left(N^{4}\right)$.

\section{Density fitted ( $\left.{ }^{\text {coul }} \mathrm{Vc}\right)$ term}

We start with the Coulomb term Eq. (37). One can notice that, due to the slow decay of the Coulomb interaction, the index- $\mathcal{J}$ summation, with the $\mathcal{B}$-vectors close to $\mathcal{J}$, is long-range, and is not affected by the locality of the exciton (i.e., the range of $(\mathcal{B}-\mathcal{J})$-vectors with significant $c_{b \mathcal{B}}^{j \mathcal{J}}$ coefficients). Since the occupied-virtual products have zero charge, the integrals decay with interorbital distance as $R^{-3}$, which implies slow but unconditional convergence in the $1 \mathrm{D}$ and $2 \mathrm{D}$ cases and just conditional convergence in the $3 \mathrm{D}$ case. Reciprocal-space density fitting ${ }^{8,41,43}$ is preferable in this case, since the need for long-range summations of the Fourier transforms are justified by the nature of the contraction. According to Eqs. (19) and (20) in Ref. 41 the Coulomb integrals from Eq. (37) are expressed as

$$
\begin{aligned}
(a \mathcal{A} i \mid j \mathcal{J} b \mathcal{B}) & =\frac{1}{n_{\mathbf{k}_{P}}} \sum_{\mathbf{k}_{P}} \exp \left(-\imath \mathbf{k}_{P} \mathbf{R}_{\mathcal{J}}\right) \\
& \times \sum_{P}(i a \mathcal{A} \mid P)\left(\mathbf{k}_{P}\right)\left(d_{P}^{j b(\mathcal{B} \ominus \mathcal{J})}\left(\mathbf{k}_{P}\right)\right)^{*},
\end{aligned}
$$

involving the Fourier images of the three-index integrals:

$$
(\text { i } a \mathcal{A} \mid P)\left(\mathbf{k}_{P}\right)=\sum_{\mathcal{P}}(i a \mathcal{A} \mid P \mathcal{P}) \exp \left({ }_{l} \mathbf{k}_{P} \mathbf{R}_{\mathcal{P}}\right),
$$

and the DF coefficients

$d_{P}^{j b(\mathcal{B} \ominus \mathcal{J})}\left(\mathbf{k}_{P}\right)=\sum_{Q}(j b(\mathcal{B} \ominus \mathcal{J}) \mid Q)\left(\mathbf{k}_{P}\right)(Q \mid P)^{-1}\left(\mathbf{k}_{P}\right)$,

with

$$
(Q \mid P)\left(\mathbf{k}_{P}\right)=\sum_{\mathcal{P}}(Q \mid P \mathcal{P}) \exp \left(\iota \mathbf{k}_{P} \mathbf{R}_{\mathcal{P}}\right)
$$

where $(P \mid Q)^{-1}\left(\mathbf{k}_{P}\right)$ denotes the inverse of the $(P \mid Q)\left(\mathbf{k}_{P}\right)$ matrix at the $\mathbf{k}$-point $\mathbf{k}_{P}$.

Now, inserting the factorization (39) into Eq. (37), changing the order of the summation, and changing the summation index $\mathcal{B}$ to $\mathcal{B}^{\prime}=\mathcal{B} \ominus \mathcal{J}$ yields

$$
\begin{aligned}
\left({ }^{\text {coul }} \mathbf{V c}\right)_{i}^{a \mathcal{A}} & =\frac{1}{n_{\mathbf{k}_{P}}} \sum_{j b \mathcal{B}^{\prime}} \sum_{\mathbf{k}_{P}} \sum_{P}(i a \mathcal{A} \mid P)\left(\mathbf{k}_{P}\right) \\
& \times\left(d_{P}^{j b \mathcal{B}^{\prime}}\left(\mathbf{k}_{P}\right)\right)^{*} c_{b \mathcal{B}^{\prime}}^{j} \sum_{\mathcal{J}} \exp \left(-\imath \mathbf{k}_{P} \mathbf{R}_{\mathcal{J}}\right),
\end{aligned}
$$

and the summation over the $\mathcal{J}$-vector completely decouples. When the number of the $\mathcal{J}$ vectors matches the chosen $\mathbf{k}_{P}$-mesh (i.e., lies within the period defined by the discrete Fourier transform, see below and Ref. 52), this summation 
reduces to $n_{\mathbf{k}_{P}} \delta_{\mathbf{k}_{P}, 0}$, which finally leads to a significant simplification for the $\left({ }^{\mathrm{Coul}} \mathbf{V c}\right)$ term:

$$
\left({ }^{\text {coul }} \mathbf{V c}\right)_{i}^{a \mathcal{A}}=\left.\left.\sum_{P}(i a \mathcal{A} \mid P)\right|_{\mathbf{k}_{P}=0} \sum_{j b \mathcal{B}^{\prime}}\left(d_{P}^{j} b \mathcal{B}^{\prime}\right)^{*}\right|_{\mathbf{k}_{P}=0} c_{b \mathcal{B}^{\prime}}^{j} .
$$

In other words, the reciprocal density fitting is now restricted to a single k-point. A similar simplification has been utilized in the density fitted calculations of the Coulomb potential in periodic AO-based HF or DFT calculations. ${ }^{53-55}$ The longrange summation over the $\mathcal{J}$-vector index is implicit, while the actual long-range contribution to the Coulomb interaction is now evaluated at the stage of the Fourier-transformation of the three- and two-index integrals Eqs. (41) and (42).

Furthermore, only the p-type GTOs of the auxiliary basis contribute to the terms with the slowest decay (s-GTO auxiliary functions are not used for the Coulomb term, vide infra). Fitting basis sets in our implementation contain only one shell of p-GTOs per center, the rest is formed by dipole-free or even completely momentless orbitals, which imply a much faster decay of the corresponding integrals. This leads to a much smaller number of long-range terms, compared to the conventional formulation, where each of the $\phi_{j} \phi_{b \mathcal{B}}$ densities contains generally a dipole moment.

\section{Density fitted (exch $\mathrm{Vc}$ ) term}

For the exchange term (38) the reciprocal fitting is possible, but not the most efficient way to proceed. In fact, in contrast to the Coulomb term, the fitted densities contain charge, which makes the Fourier lattice summations (41) and (42) conditionally convergent already for the 1D case. At the same time, the actual contraction in Eq. (38) is short range, provided the exciton is at least moderately localized (for very delocalized excitons the local direct space approach is not efficient anyway). Indeed, the actual summation range for the $\mathcal{J}$-index is dictated by the overlap between the Wannier functions, and for the $\mathcal{B}$-index additionally by the decay rate of the CIS coefficients $c_{b \mathcal{B}^{\prime}}^{j}$.

The local direct-space density fitting ${ }^{43,56,57}$ scheme is more appropriate in this case. In the present implementation we use a unique fit-domain $\mathrm{D}_{\text {fit }}$ for all the two-electron integrals involved in the exchange term which allows us to use the one-term robust density fitting ${ }^{51,57}$

$$
(j \mathcal{J} i \mid a \mathcal{A} b \mathcal{B})=\sum_{P \mathcal{P} \in \mathrm{D}_{\mathrm{fit}}} d_{P \mathcal{P}}^{i j \mathcal{J}}(P \mathcal{P} \mid a \mathcal{A} b \mathcal{B}),
$$

with

$$
d_{P \mathcal{P}}^{i j \mathcal{J}}=\sum_{Q \mathcal{Q} \in \mathrm{D}_{\mathrm{fit}}}(i j \mathcal{J} \mid Q \mathcal{Q})(Q \mathcal{Q} \mid P \mathcal{P})^{-1} .
$$

Now $(Q \mathcal{Q} \mid P \mathcal{P})^{-1}$ is the inverse of the square metric matrix within the $\mathrm{D}_{\text {fit }}$-domain. For the unique fit-domain only a single matrix inversion has to be carried out. At the same time, the fit-domain should be sufficiently large to be able to provide support for all the $\phi_{i} \phi_{j \mathcal{J}}$ and $\phi_{a \mathcal{A}} \phi_{b \mathcal{B}}$ product densities appearing in the calculation. For polymers it is generally not problematic to fulfill this condition. However, for 2D or 3D systems it can become critical, since the matrix to be inverted might become very large. Work is in progress on implementing the density-specific fit-domain technique, similar to that of Refs. 43 and 58, which circumvents this problem, but requires a three-term robust density fitting formalism. ${ }^{51}$

The expression for the exchange term with the local density fitting approximation takes the form

$$
\begin{aligned}
\left({ }^{\text {exch }} \mathbf{V c}\right)_{i}^{a \mathcal{A}}= & \sum_{j \mathcal{J}} \sum_{P \mathcal{P} \in \mathrm{D}_{\text {fit }}} d_{P \mathcal{P}}^{i j \mathcal{J}} \sum_{b \mathcal{B}} c_{b(\mathcal{B} \ominus \mathcal{J})}^{j} \\
& \times(P(\mathcal{P} \ominus \mathcal{J}) \mid a(\mathcal{A} \ominus \mathcal{J}) b(\mathcal{B} \ominus \mathcal{J})) \\
= & \sum_{j \mathcal{J}} \sum_{P \mathcal{P} \in \mathrm{D}_{\text {fit }}} d_{P \mathcal{P}}^{i j \mathcal{J}} \sum_{b \mathcal{B}^{\prime}} c_{b \mathcal{B}^{\prime}}^{j} \\
& \times\left(P(\mathcal{P} \ominus \mathcal{A}) \mid a b\left(\mathcal{B}^{\prime} \ominus \mathcal{A} \oplus \mathcal{J}\right)\right) .
\end{aligned}
$$

Here, we used the translational invariance of the integrals and the CIS coefficients and again redefined the $\mathcal{B}$ vector.

\section{IMPLEMENTATION}

\section{A. Introduction of a k-mesh and the supercell}

Since in practical calculations analytic integration of the Brillouin zone is not feasible, one has to introduce a finite $\mathbf{k}$-mesh for the numerical integration through the discrete Fourier transform. The number of k-points of the mesh needed for the calculation is not known a priori and therefore set as an input parameter.

In fact, the density of the mesh, sufficient for the calculation is strongly dependent on the locality of the exciton in the direct space. The back-Fourier-transform Eq. (36) of the CIS coefficients $(\mathbf{c})_{a}^{i}(\mathbf{k})$ with a finite number of $\mathbf{k}$-points generates a periodic direct space image of it, with a period forming a supercell (sc) with a size exactly matching the number of the k-points. The physically relevant part of the exciton is located in the zero Wigner-Seitz supercell, since its periodically repeated images are artifacts of the finiteness of the $\mathbf{k}$-mesh. ${ }^{41,52}$ Therefore, in our approach we always restrict the range for the $\mathcal{A}$ vectors of the CIS coefficients $(\mathbf{c})_{a \mathcal{A}}^{i}$, or the trial vectors $(\Delta \mathbf{c})_{a \mathcal{A}}^{i}$ to this supercell, setting the values outside the supercell to zero:

$$
(\mathbf{c})_{a \mathcal{A}}^{i}= \begin{cases}\frac{1}{\sqrt{n_{\mathbf{k}}}} \sum_{\mathbf{k}} \exp \left(\imath \mathbf{k} \mathbf{R}_{\mathcal{A}}\right)(\mathbf{c})_{a}^{i}(\mathbf{k}) & \text { if } \mathcal{A} \in(\mathrm{sc}), \\ 0 & \text { if } \mathcal{A} \notin(\mathrm{sc}) .\end{cases}
$$

Furthermore, as is well known from the discrete Fouriertransform theory, it can be considered as an accurate numerical quadrature only if the resulting function goes to zero at the borders of the supercell, defined by the given $\mathbf{k}$-mesh, or in other words, is localized within this supercell. This implies that the number of k-points needed for the correct description of an exciton strongly depends on its nature: the more local it is the less k-points in the reciprocal representation of the CIS coefficients are needed.

Next, even if the CIS coefficients are restricted to the supercell, the range of nonzero matrix-vector products $(\mathbf{V c})_{i}^{a \mathcal{A}}$, which also depends on the sparsity of the Hamiltonian, can go beyond this range. This might violate the invariance of the small h-matrix (12) with respect to the representation 
(direct or reciprocal) of $\Delta \mathbf{c}$ and $\mathbf{H} \Delta \mathbf{c}$. Consider the small $\mathbf{h}^{\text {(dir) }}$-matrix, defined in Eq. (12), evaluated in the direct space:

$$
h_{r s}^{(\mathrm{dir})}=\sum_{i a \mathcal{A}}\left(\Delta \mathbf{c}^{\dagger(r)}\right)_{a \mathcal{A}}^{i}\left(\mathbf{H} \Delta \mathbf{c}^{(s)}\right)_{i}^{a \mathcal{A}} .
$$

The $\mathcal{A}$-vectors in $(\mathbf{H} \Delta \mathbf{c})_{i}^{a \mathcal{A}}$ outside the supercell do not contribute to the matrix $\mathbf{h}^{\text {(dir) }}$, since the matching CIS coefficients are zero beyond the supercell by construction (48). However, if the $\mathbf{h}$-matrix is calculated in the reciprocal space employing the vector $(\mathbf{H} \Delta \mathbf{c})(\mathbf{k})$ (as is done in our approach), the inclusion of the outer tails of the $(\mathbf{H} \Delta \mathbf{c})_{i}^{a \mathcal{A}}$ in the Fourier transform (29), would have an effect on the result. Indeed, according to Eqs. (12) and (29) one obtains

$$
\begin{aligned}
h_{r s}^{(\mathrm{recipr})}(\mathbf{k})= & \sum_{i a \mathbf{k}}\left(\Delta \mathbf{c}^{\dagger(r)}\right)_{a}^{i}(\mathbf{k})\left(\mathbf{H} \Delta \mathbf{c}^{(s)}\right)_{i}^{a}(\mathbf{k}) \\
= & \sum_{i a \mathbf{k}}\left(\Delta \mathbf{c}^{\dagger(r)}\right)_{a}^{i}(\mathbf{k}) \\
& \times\left[\frac{1}{\sqrt{n_{\mathbf{k}}}} \sum_{\mathcal{A}} \exp \left(-l \mathbf{k} \mathbf{R}_{\mathcal{A}}\right)\left(\mathbf{H} \Delta \mathbf{c}^{(r)}\right)_{i}^{a \mathcal{A}}\right] .
\end{aligned}
$$

The summation range of the index $\mathcal{A}$ can be separated in $\mathcal{A} \in(\mathrm{sc})$ and $\mathcal{A} \notin(\mathrm{sc})$. The former sum constitutes the direct space matrix $h^{(\mathrm{dir})}$, whereas the latter appears as a generally nonzero term in the equation:

$$
\begin{aligned}
h_{r s}^{(\mathrm{recipr})}(\mathbf{k})= & h_{r s}^{(\mathrm{dir})}+\frac{1}{\sqrt{n_{\mathbf{k}}}} \sum_{\mathcal{A} \notin(s c)} \sum_{i a}\left(\mathbf{H} \Delta \mathbf{c}^{(r)}\right)_{i}^{a \mathcal{A}} \\
& \times \sum_{\mathbf{k}} \exp \left(-\imath \mathbf{k} \mathbf{R}_{\mathcal{A}}\right)\left(\Delta \mathbf{c}^{\dagger(r)}\right)_{a}^{i}(\mathbf{k}) .
\end{aligned}
$$

This shows that the $\mathbf{h}^{\text {(recipr) }}$-matrix, evaluated in the reciprocal space, differs from $\mathbf{h}^{(\mathrm{dir})}$, if the elements of $(\mathbf{H} \Delta \mathbf{c})_{i}^{a \mathcal{A}}$ beyond the supercell are included in the Fourier transform. In our method we calculate the two-electron part $(\mathbf{V} \Delta \mathbf{c})_{i}^{a \mathcal{A}}$ of the full vector $(\mathbf{H} \Delta \mathbf{c})_{i}^{a \mathcal{A}}$ in the direct space and then transform it to the reciprocal space. In order to enforce the invariance of the h-matrix, the values of $(\mathbf{V} \Delta \mathbf{c})_{i}^{a \mathcal{A}}$ beyond the supercell are not included in the Fourier transform (29) (and essentially not computed), thus making the second summand in Eq. (51) zero.

\section{B. The norm of the CIS vector}

Since the Davidson diagonalization procedure is carried out in the reciprocal space each new trial vector is orthogonalized to the previous ones and normalized to unity, which also guarantees the normality of the actual CIS vectors in the reciprocal space. The reciprocal space images of the local CIS coefficients are also normalized,

$$
N^{(\text {recipr/local })}=\sum_{i a b \mathbf{k}} c_{a}^{i *}(\mathbf{k}) S_{a b}^{(\mathrm{PAO})}(\mathbf{k}) c_{b}^{i}(\mathbf{k})=1 .
$$

The direct space norm of the CIS vectors

$$
\begin{aligned}
N^{(\mathrm{dir})} & =\sum_{i a b} \sum_{\mathcal{A B}} c_{a \mathcal{A}}^{i *} S_{a \mathcal{A} b \mathcal{B}}^{(\mathrm{PAO})} c_{b \mathcal{B}}^{i} \\
& =\sum_{i a b} \sum_{\mathcal{A B}} c_{a \mathcal{A}}^{i *} S_{a b(\mathcal{B} \ominus \mathcal{A})}^{(\mathrm{PAO})} c_{b \mathcal{B}}^{i},
\end{aligned}
$$

however, might differ from unity. Indeed, rewriting Eq. (53) via Fourier transforms of the reciprocal images of the involved quantities and restricting the values to the supercell yields

$$
\begin{aligned}
N^{(\mathrm{dir})}= & \frac{1}{n_{\mathbf{k}}^{2}} \sum_{i a b} \sum_{\mathbf{k k}^{\prime} \mathbf{k}^{\prime \prime}} c_{a}^{i *}(\mathbf{k}) S_{a b}^{(\mathrm{PAO})}\left(\mathbf{k}^{\prime}\right) c_{b}^{i}\left(\mathbf{k}^{\prime \prime}\right) \\
& \times \sum_{A \in(\mathrm{sc})} \exp \left(-\imath \mathbf{k} \mathbf{R}_{\mathcal{A}}\right) \\
& \times \sum_{\mathcal{B} \in(\mathrm{sc})} \exp \left(-\imath \mathbf{k}^{\prime}\left(\mathbf{R}_{\mathcal{B}}-\mathbf{R}_{\mathcal{A}}\right)\right) \exp \left(\iota \mathbf{k}^{\prime \prime} \mathbf{R}_{\mathcal{B}}\right) . \\
& \forall(\mathcal{B} \ominus \mathcal{A}) \in(\mathrm{sc})
\end{aligned}
$$

Here we used expression (36) and the inverse of the transformation (23). In the last sum of (54) not all vectors $\mathcal{B}$ from the supercell are included, but only those, which for a given $\mathcal{A}$ fulfill the condition $(\mathcal{B} \ominus \mathcal{A}) \in(\mathrm{sc})$, since otherwise the backFourier transform would generate artificial periodic images of the overlap matrix. This truncation actually destroys the direct link to the reciprocal norm (52). In order to re-establish this connection we subtract and add the missing terms:

$$
\begin{aligned}
& N^{(\text {direct } / \text { local })}= \frac{1}{n_{\mathbf{k}}^{2}} \sum_{i a b} \sum_{\mathbf{k} \mathbf{k}^{\prime} \mathbf{k}^{\prime \prime}} c_{a}^{i *}(\mathbf{k}) S_{a b}^{(\mathrm{PAO})}\left(\mathbf{k}^{\prime}\right) c_{b}^{i}\left(\mathbf{k}^{\prime \prime}\right) \\
& \times \sum_{A \in(\mathrm{sc})} \exp \left(-l\left(\mathbf{k}-\mathbf{k}^{\prime}\right) \mathbf{R}_{\mathcal{A}}\right) \\
& \times\left[\sum_{\mathcal{B} \in(\mathrm{sc})} \exp \left(-l\left(\mathbf{k}^{\prime}-\mathbf{k}^{\prime \prime}\right) \mathbf{R}_{\mathcal{B}}\right)\right. \\
&\left.-\sum_{\mathcal{B} \in(\mathrm{sc})} \exp \left(-l\left(\mathbf{k}^{\prime}-\mathbf{k}^{\prime \prime}\right) \mathbf{R}_{\mathcal{B}}\right)\right] \\
& \forall(\mathcal{B} \ominus \mathcal{A}) \\
&= \sum_{i a b \text { (sc) }} \sum_{\mathbf{k} \mathbf{k}^{\prime} \mathbf{k}^{\prime \prime}} c_{a}^{i *(\mathbf{k}) S_{a b}^{(\mathrm{PAO})}\left(\mathbf{k}^{\prime}\right) c_{b}^{i}\left(\mathbf{k}^{\prime \prime}\right) \delta_{\mathbf{k}^{\prime \prime}, \mathbf{k}^{\prime}} \delta_{\mathbf{k}^{\prime}, \mathbf{k}}} \\
&-\sum_{A \in(\mathrm{sc})} c_{a \mathcal{A}}^{i *} \sum_{\mathcal{B} \in \mathrm{sc}} S_{a b(\mathcal{B} \ominus \mathcal{A})}^{(\mathrm{PAO})} c_{b \mathcal{B}}^{i} . \\
& \forall(\mathcal{B} \ominus \mathcal{A}) \notin \mathrm{sc}
\end{aligned}
$$

The first term is actually the reciprocal norm, Eq. (52), i.e., 1 , but the subtrahend is not necessarily zero. We note that, since both $\mathcal{B}$ and $\mathcal{A}$ belong to the supercell, their difference, which for this term is outside the supercell, can be brought there by adding or subtracting the supercell translation vector $\mathbf{R}_{\mathrm{sc}}$. Since for any $\mathbf{k}$ point from the chosen mesh $\exp \left( \pm \imath \mathbf{k} \mathbf{R}_{\mathrm{sc}}\right)=1$, this exponential can be harmlessly multiplied to the back Fourier transform of the overlap matrix $S_{a b}^{(\mathrm{PAO})}\left(\mathbf{k}^{\prime}\right)$, such that $S_{a b(\mathcal{B} \ominus \mathcal{A})}^{(\mathrm{PAO})}$ is replaced by the ma$\operatorname{trix} S_{a b\left(\mathcal{B} \ominus \mathcal{A} \pm \mathbf{R}_{\mathrm{sc}}\right)}^{(\mathrm{PAO})}$. This simplifies the expression for the direct norm to

$$
N^{(\text {direct/local })}=1-\sum_{A \in(\mathrm{sc})} c_{a \mathcal{A}}^{i *} \sum_{\substack{\mathcal{B} \in(\mathrm{sc}) \\ \forall\left(\mathcal{B} \ominus \mathcal{A} \pm \mathbf{R}_{\mathrm{sc}}\right) \\ \in(\mathrm{sc})}} S_{a b\left(\mathcal{B} \ominus \mathcal{A} \pm \mathbf{R}_{\mathrm{sc}}\right)}^{(\mathrm{PAO})} c_{b \mathcal{B}}^{i}
$$


Now, due to the locality of the PAO overlap matrix an element $S_{a b\left(\mathcal{B} \ominus \mathcal{A} \pm \mathbf{R}_{\mathrm{sc}}\right)}^{(\mathrm{PAO}}$ is non-negligible only if the vector $\mathbf{R}_{\mathcal{B}}$ $-\mathbf{R}_{\mathcal{A}} \pm \mathbf{R}_{\mathrm{sc}}$ is small (i.e., close to the reference cell). Since both vectors $\mathcal{A}$ and $\mathcal{B}$ are restricted to the Wigner-Seitz supercell, this happens only if both of them are located at its edges (actually at the opposite sides of it). If the exciton is well localized within the supercell, the CIS coefficients $c_{a \mathcal{A}}^{i}$ and $c_{b \mathcal{B}}^{i}$ are small at the edges, which makes the second term in Eq. (56) close to zero, and the direct space norm to unity. In other words, when the chosen $\mathbf{k}$-mesh is adequate for describing the exciton, the direct norm is close to the reciprocal one. This property suggests that the direct space norm can be used as an indicator for the reliability of the chosen $\mathbf{k}$-mesh. This issue is illustrated in Sec. IV on a set of test calculations.

\section{Evaluation of the transformation matrices}

The implemented CIS method needs the underlying Hartree-Fock ground state solution, which is provided by the CRYSTAL program. ${ }^{48}$ Namely, it delivers the Fock matrix, and the information on the structural parameters, basis set and the symmetry. In addition, CRYSTAL generates Wannier functions according to the Wannierization-localizationsymmetrization procedure by Zicovich-Wilson, ${ }^{46}$ Casassa $^{59}$ and co-workers. The constructed Wannier functions are represented via the $\mathrm{AO}$ expansion coefficients.

Using these quantities it is possible to evaluate the transformation matrices $\mathbf{W}(\mathbf{k}), \mathbf{Q}(\mathbf{k})$, and $\overline{\mathbf{Q}}(\mathbf{k})$ (see Sec. II D 1) and the explicit direct space AO representation of the PAOs, needed for the calculation of the Vc-terms (see Sec. II E). First, the AO Fock matrix, overlap matrix and WF coefficients are Fourier transformed to the reciprocal space. Using the latter, the Fock matrix can be transformed to the basis of the Fourier images of the Wannier functions. The eigenvectors of this matrix at each $\mathbf{k}$-point form the transformation matrices $\mathbf{W}^{\dagger}(\mathbf{k})$ [Eq. (18)].

Next, the projector from the occupied manifold [Eq. (19)] is also constructed in the reciprocal space:

$$
\begin{aligned}
1-\hat{P}(\mathbf{k}) & =1-\sum_{\bar{i}}\left|\phi_{\bar{i}}^{\mathrm{CAN}}(\mathbf{k})\right\rangle\left\langle\phi_{\bar{i}}^{\mathrm{CAN}}(\mathbf{k})\right| \\
& =\sum_{\bar{a}}\left|\phi_{\bar{a}}^{\mathrm{CAN}}(\mathbf{k})\right\rangle\left\langle\phi_{\bar{a}}^{\mathrm{CAN}}(\mathbf{k})\right|,
\end{aligned}
$$

and used to calculate the reciprocal images of the PAOs, which are then back-Fourier-transformed to the direct space. $^{60}$

From Eqs. (57) and (19) it follows that the reciprocal space AO expansion coefficients $C_{\mu a}^{\mathrm{PAO}}(\mathbf{k})$ of the PAOs can be expressed via the $\mathrm{AO}$ coefficients of canonical virtual orbitals $C_{\mu \bar{a}}^{\mathrm{CAN}}(\mathbf{k})$ as

$$
C_{\mu a}^{\mathrm{PAO}}(\mathbf{k})=\left.\sum_{\bar{a} \mu^{\prime}} C_{\mu \bar{a}}^{\mathrm{CAN}}(\mathbf{k}) C_{\mu^{\prime} \bar{a}}^{\mathrm{CAN} \dagger}(\mathbf{k}) S_{\mu^{\prime} \nu}^{\mathrm{AO}}(\mathbf{k})\right|_{a=\nu} .
$$

Now, expressing the orbitals on the right- and left-handside of Eq. (21) via their AO expansion, and using Eq. (58) yields the formula for the practical evaluation of the $\mathbf{Q}(\mathbf{k})$ transformation:

$$
Q_{\bar{a} a}(\mathbf{k})=\left.\sum_{\mu} C_{\bar{a} \mu}^{\mathrm{CAN} \dagger} S_{\mu \nu}^{\mathrm{AO}}(\mathbf{k})\right|_{a=\nu} \cdot
$$

As already mentioned in Sec. II D 1 the basis of the reciprocal images of the PAOs is redundant. Indeed, the number of the PAOs so-obtained at each $\mathbf{k}$-point is equal to the number of AOs, they are constructed from, while the actual virtual space has a dimensionality lower than that of the AOs by the number of the occupied states at a given $\mathbf{k}$-point. The pseudoinverse $\mathbf{X}(\mathbf{k})$ of the $\mathbf{S}^{\mathrm{PAO}}(\mathbf{k})$ matrix is constructed via its singular value decomposition, namely, by diagonalizing the $\mathbf{S}^{\mathrm{PAO}}(\mathbf{k})$ matrix, inverting its nonzero eigenvalues and back-transforming this diagonal matrix. The $\overline{\mathbf{Q}}(\mathbf{k})$-transformation is then generated according to Eq. (25).

\section{Density fitting and the ranges for lattice summations}

For the correct density fitting treatment of the Coulomb (44) and exchange (47) terms the ranges for various vector indices are to be determined. In the Coulomb term [Eqs. (40)-(42) and (44)] these are the indices $\mathcal{P}$ and $\mathcal{B}$. The range for the latter index can easily be prescreened. It, first, should not go beyond the supercell, since the contraction along this index with the CIS coefficients outside the supercell is zero by construction. And, second, it should be sufficiently close to the reference cell, since otherwise the corresponding three-index integral or fitting coefficient would be negligible.

The summations involving the $\mathcal{P}$-index in Eqs. (41) and (42) are more delicate. As mentioned above, the fitted densities are chargeless, which implies a slow but absolute $1 / R^{3}$ convergence of the Fourier series in the $1 \mathrm{D}$ case. In order to maintain this convergence rate also the auxiliary functions have to be chargeless. This can be elegantly achieved by using combined GTO/Poisson auxiliary basis sets. ${ }^{41,43,61}$ The main body of the fitting basis is formed by the momentless Poisson type-orbitals (PTOs) - Laplacians of GTOs. ${ }^{62,63}$ Coulomb integrals with PTOs reduce to one-electron overlap or kinetic energy integrals, which are easier to calculate and, most importantly, decay exponentially with the interorbital distance. This property makes the vast majority of the three- and two-index Coulomb integrals essentially short range.

Since the momentless Poisson-type fitting functions alone cannot describe the true densities, the auxiliary basis sets are augmented by a few GTOs, by one shell for each angular momentum (up to g-type) per center. Since the fitted densities in the Coulomb term are chargeless, s-GTOs, causing the most problematic divergent terms, are not needed. In the 1D case the Fourier series (41) and (42), involving all the included auxiliary GTOs, converge. The evaluations and Fourier summations of the three- and two-index integrals are performed on the fly until the values of the integrals drop below a certain threshold $\left(10^{-7}\right.$ by default). Due to the small number of auxiliary GTOs and the need for only one k-point in the Coulomb density fitting, this is an inexpensive procedure. In higher dimensionalities the convergence rate of these Fourier series will slow down, and the number of the integrals to be evaluated will grow considerably. In this case the mul- 
tipole expansion of the integrals combined with convergence acceleration techniques like the Ewald procedure will be required.

The density fitting procedure used for the exchange term, as is seen from Eq. (47), is quite different. First, the local fitting scheme is used, which implies an a priori truncation of the auxiliary space to a fit-domain. For the robust fitting the fit-domain should cover all the fitted densities, in our case it is $\phi_{i} \phi_{j \mathcal{J}}$ and $\phi_{a \mathcal{A}} \phi_{b \mathcal{B}}$. Due to the locality of the Wannier functions the integrals involving the $\phi_{i} \phi_{j \mathcal{J}}$ densities quickly fade to zero with increasing $\mathcal{J}$. Therefore, the range for this index can be prescreened by comparing the products of the WFs LCAO coefficients with a certain threshold.

The densities $\phi_{a \mathcal{A}} \phi_{b \mathcal{B}}$ possess similar decay properties, now depending on the length of the $\mathcal{B} \ominus \mathcal{A}$ vectors, which can be prescreened. The restriction of the $\mathcal{A}$-vector to the supercell (see Sec. III A) determines the range for the possible values of the $\mathcal{B}$-vector. This range can be further reduced by taking into account the contraction of the integral with the CIS vectors $c_{b \mathcal{B}}^{j \mathcal{J}}$ (38). The latter quantity is zero if the vector $\mathcal{B} \ominus \mathcal{J}$ is outside the supercell. Then using the prescreened range for $\mathcal{J}$, one can sort out the un-needed $\mathcal{B}$-vectors. This range for the $\mathcal{B}$-vectors, which is the most extended among those considered here, determines the range for the $\mathcal{P}$ vectors of the fit-domain.

Analogously to the Coulomb term, a combined PTO/GTO fitting basis set is used for the fitting of the exchange term. Although the property of the exponential decay of the PTO-based integrals is here of less relevance, since the range of the fitting functions is anyway restricted to the fit domain, the savings in the integral generation are still significant. In contrast to the Coulomb-part, the fitted densities of the exchange-integrals do possess charges, which requires inclusion of s-GTO auxiliary functions. Since evaluation of three-index integrals with such functions within the fit-domain is relatively inexpensive, the s-type-orbital part of the auxiliary basis for the exchange term is represented solely by GTOs.

In the evaluation of both the ${ }^{\text {coul }} \mathbf{V} \mathbf{c}$ and ${ }^{\text {exch }} \mathbf{V}$ terms, the contraction of the ket-side three-index integrals with the CIS coefficients is done first, as is shown in Eqs. (44) and (47). Subsequently the result of this contraction, which has a much smaller size than the initial three-index integral, is contracted with the DF-coefficients. This order of matrix multiplications, which scales nominally as $\mathcal{O}\left(N^{4}\right)$, explicitly avoids the expensive $\mathcal{O}\left(N^{5}\right)$-scaling assembly of the four-index integrals.

The actual scaling of the computational cost of the method can be considered relative to two parameters: the size of the unit cell, and the number of k-points, which defines the size of the supercell. The latter quantity essentially depends on the locality of the exciton and is not known a priori. Since the ranges of both the excitonic $\mathcal{A}$ and auxiliary $\mathcal{P}$ lattice vectors are affected by the increase of the supercell, the contractions in Eq. (47) scale quadratically with its size. On the other hand, when the unit cell size is increased, the computational cost scales only linearly, since the supercell size can then proportionally be decreased, provided that the locality of the exciton under consideration remains unchanged. If this is not the case, then the linear scaling with respect to cell size is weighted with the quadratic scaling due to the additional increase or decrease of the spread of the exciton. This analysis shows that the method is more sensitive to the diffuseness of the exciton than to the number of atoms in the unit cell. A further reduction of the former quadratic to linear scaling is possible when local-density-fitting schemes ${ }^{43,56,58}$ are applied.

\section{E. The initial guess for the Davidson procedure}

Since the cost and correctness of the local scheme, as described above, essentially depends on the locality of the exciton, the starting guess for the Davidson procedure has to be chosen with care. Under such circumstances it is natural to use a direct-space defined starting guess, which can be constructed to be local. We have investigated three types of direct space starting guesses and one in reciprocal space:

1. Excitations from all WFs to all PAOs within the zero cell with an equal weight. This simple starting vector is well localized, but depends on the choice of the origin of the unit cell and favors totally symmetric excitons (with respect to the point group symmetry of the center of the unit cell).

2. Equiweighted excitations from the zero-cell WFs onto the PAOs within their Boughton-Pulay PAO-domains. ${ }^{40}$ Such a vector is even more localized than in the previous case and at the same time does not depend on the choice of the origin. However, it is still totally symmetric, which can be inadequate for an exciton with some other symmetry.

3. Several starting vectors corresponding to smallest values of the differences between the diagonal elements of the Fock matrix in PAO/WF basis. This mimics HOMOLUMO-like excitations, yet in direct space. The starting vectors can be of different symmetries, which makes it more flexible than the previous two cases. However, there is no guarantee that the vectors chosen in such a manner would contribute significantly to the lowest exciton. If not, this starting guess, which uses several starting vectors rather than just one, might increase the cost of the calculations without a significant gain in the convergence.

4. The staring vector in the reciprocal space with the equiweighted vertical excitations for all k-points from the highest occupied band to the lowest virtual band. Since we are focusing on the lowest-energy exciton, these are the excitations which are expected to contribute the most. The symmetry of the exciton is also likely to be correctly described by these excitations. However, the locality of the starting vector transformed to the direct space might be lost. In fact, the convergence of the Fourier series is critically affected by discontinuities in the Fourier-expanded function. As was shown by Kohn ${ }^{64}$ for $1 \mathrm{D}$ and des Cloizeaux ${ }^{65}$ for $3 \mathrm{D}$ cases on the example of the Fourier transforms connecting Bloch and Wannier functions, the exponential decay of the latter can be achieved only if the corresponding band is separated 
from the other bands by energy gaps. In our context, the Fourier-transform of an improperly chosen reciprocal trial vector looses its localization in the direct space, which, when exceeding the supercell, can become troublesome for our method. Even when excitations from the whole band are included in the reciprocal starting guess, making it a smooth function of $\mathbf{k}$, a possible intersection with other bands can lead to nonanalyticity ${ }^{64}$ and, thus, to a poor localization in the direct space. Probably, such intersections are unlikely in the 1D case and, as the calculations have shown, this reciprocal starting guess is the most efficient for the systems studied here (see Sec. IV). However, in 3D systems, where the lower conduction band as a rule crosses with higher ones, this might no longer be the case.

\section{CALCULATIONS}

The method described above has been applied to several $1 \mathrm{D}$ test systems. In these calculations we investigate the efficiency and reliability of the method, in particular the convergence with respect to the number of k-points, the accuracy of the density fitting approximation, etc. We have considered van-der-Waals $(\mathrm{Ar}), \mathrm{H}$-bonded $\left(\mathrm{H}_{2} \mathrm{O}\right)$, ionic $(\mathrm{LiH}) 1 \mathrm{D}$ chains and several "real" covalent polymers: polyethylene, polystyrene, and polybiphenyl. Some of the studied systems do not exist in 1D form in reality, but can serve as prototypes for 3D solids and provide information on the locality of excitons, and thus the feasibility of the local scheme, for such crystals. Table I compiles the results of the CIS calculations for singlet and triplet $\Gamma$-point excitons. The geometries of the covalent polymers have been optimized with the CRYSTAL $\operatorname{code}^{48}$ at the DFT/B3LYP level, for the other systems a 1D cut from the 3D solid was taken. Some of the studied systems are sketched in Fig. 1, and all the structural data are given explicitly in the supplementary material. ${ }^{66}$

The numbers of $\mathbf{k}$-points reported in Table I corresponds to the converged (with respect to this parameter) energies. This is one of the key parameters, since it reflects the locality of the excitons. One can see that in the weakly bound argon polymer, as anticipated, the excitons are rather local. In

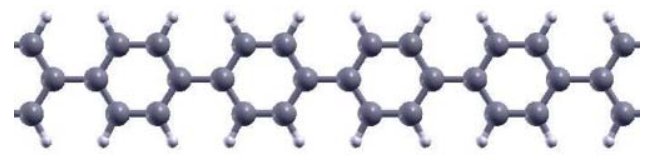

(i) parallel polybiphenyl

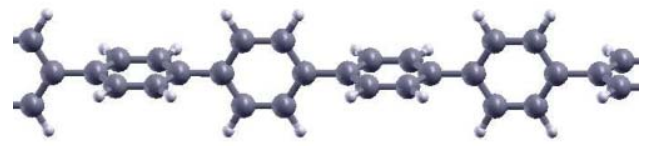

(ii) perpendicular polybiphenyl

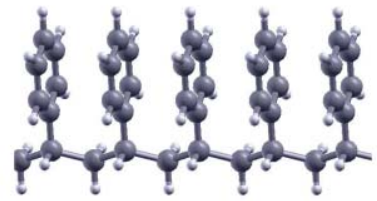

(iii) isotactic polystyrene

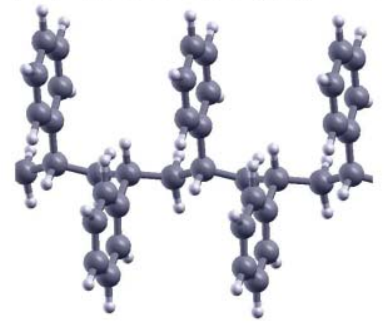

(iv) syndiotactic polystyrene

FIG. 1. Maximal CIS coefficients per cell (singlet state) for (i) $\mathrm{LiH}$ and (ii) biphenyl (solid: parallel; dashed: perpendicular).

the ionic $\mathrm{LiH}$ and $\mathrm{H}$-bonded water polymers they are somewhat less localized (note the "doubled" unit cell in the latter system consisting of two molecular units). However, neither of these excitons can be considered as delocalized, so the $\mathbf{k}$ meshes needed in such systems and the corresponding direct space supercells can be processed without big effort, presumably, also in the 3D case. On the other hand, in the covalent systems studied here the locality of the exciton depends essentially on the structure and the type of the excitation. In

TABLE I. Number of atoms per unit cell ( $\left.\mathrm{n}_{\text {atoms }}\right)$, Hartree-Fock HOMO-LUMO band gaps $\left(\mathrm{E}_{\mathrm{g}}\right)$, negatives of the highest occupied orbital energies $\left(-\epsilon_{\mathrm{HOMO}}\right)$, number of k-points used, vertical excitation energies to the lowest singlet and triplet exciton state. The fitting basis set related to the cc-pVDZ AO basis was used. The elapsed times were measured on a single core Intel Xeon E5450 @ $3.00 \mathrm{GHz}$.

\begin{tabular}{|c|c|c|c|c|c|c|c|c|c|}
\hline \multirow[b]{2}{*}{ System } & \multirow[b]{2}{*}{$\mathrm{n}_{\text {atoms }}$} & \multirow[b]{2}{*}{$\mathrm{E}_{\mathrm{g}} / \mathrm{eV}$} & \multirow[b]{2}{*}{$-\epsilon_{\mathrm{HOMO}} / \mathrm{eV}$} & \multicolumn{3}{|c|}{ Singlet } & \multicolumn{3}{|c|}{ Triplet } \\
\hline & & & & $n_{\mathbf{k}}$ & $\mathrm{E}_{\mathrm{sin}} / \mathrm{eV}$ & Time & $n_{\mathbf{k}}$ & $\mathrm{E}_{\mathrm{tri}} / \mathrm{eV}$ & Time \\
\hline $\mathrm{Ar}^{\mathrm{a}}$ & 1 & 30.864 & 15.555 & 5 & 21.734 & $5 \mathrm{~s}$ & 4 & 20.519 & $3 \mathrm{~s}$ \\
\hline $\mathrm{LiH}^{\mathrm{a}}$ & 2 & 11.485 & 9.269 & 8 & 6.606 & $18 \mathrm{~s}$ & 8 & 5.502 & $16 \mathrm{~s}$ \\
\hline $\mathrm{H}_{2} \mathrm{O}^{\mathrm{a}, \mathrm{c}}$ & 6 & 18.834 & 13.172 & 5 & 10.378 & $54 \mathrm{~s}$ & 3 & 9.485 & $21 \mathrm{~s}$ \\
\hline All-trans $-\mathrm{C}_{2} \mathrm{H}_{4}{ }^{\mathrm{a}}$ & 6 & 16.996 & 10.902 & 11 & 11.748 & $\sim 11 \mathrm{~min}$ & 6 & 10.547 & $\sim 4 \min$ \\
\hline Biphenyl $(\|)^{\mathrm{b}}$ & 11 & 7.754 & 6.497 & 14 & 3.835 & $\sim 100 \mathrm{~min}$ & 11 & 2.192 & $\sim 60 \mathrm{~min}$ \\
\hline Biphenyl $(\perp)^{\mathrm{b}, \mathrm{c}}$ & 22 & 12.301 & 8.860 & 3 & 6.385 & $\sim 40 \min$ & 3 & 3.274 & $\sim 35 \mathrm{~min}$ \\
\hline Styrene (iso) ${ }^{\mathrm{b}}$ & 16 & 10.863 & 5.518 & 5 & 6.454 & $\sim 80 \mathrm{~min}$ & 5 & 3.199 & $\sim 50 \mathrm{~min}$ \\
\hline Styrene (syn) $)^{b, c}$ & 32 & 12.281 & 8.141 & 3 & 6.350 & $\sim 3 \mathrm{~h} 40 \mathrm{~min}$ & 3 & 3.316 & $\sim 2 \mathrm{~h} 30 \mathrm{~min}$ \\
\hline
\end{tabular}

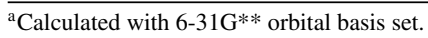

${ }^{\mathrm{b}}$ Calculated with 6-31G orbital basis set with upscaled exponent of the most diffuse sp-function of carbon from 0.16871448 to 0.18 .

${ }^{\mathrm{c}}$ The unit cell includes two molecular units. 
the case of polyethylene the lowest-energy exciton, which spreads out along the $\mathrm{C}-\mathrm{C}$ bonds, is noticeably less localized than in polystyrene, where it corresponds to a $\pi \rightarrow \pi^{*}$-type exciton well localized on the ring. In polybiphenyl with parallel orientation of the rings the same $\pi \rightarrow \pi^{*}$-exciton can essentially delocalize. At the same time, the $90^{\circ}$-orientation of the rings in the perpendicular polybiphenyl blocks this $\pi$ type delocalization and the exciton becomes well localized.

Apparently, there exists a link between the value of the excitation energy and the locality of the exciton. Indeed, the exciton in the argon chain, which has the largest HOMOLUMO difference and highest excitation energy among the systems studied here, is the most localized. Similarly, the low excitation energy in parallel polybiphenyl corresponds to a relatively delocalized exciton. However, this tendency is not unequivocal, since also short-range effects can influence the excitation energy significantly. Indeed, the excitons in $\mathrm{LiH}$, and especially polystyrene are rather local and at the same time considerably less energetic than the much more delocalized exciton of polyethylene.

The Coulomb term (44), which is present in the CIS Hamiltonian only for the singlet states, is well known to produce a destabilizing contribution. This effect is reproduced in our calculations, where the triplet excitation energies are always lower than the corresponding singlet ones, particularly so for $\pi \rightarrow \pi^{*}$-type of the excitation. Additionally, the Coulomb term might facilitate a further delocalization of the exciton, since in some (but not all) cases the triplet excitons are noticeably more localized. Similar effects have been observed in local CC2 calculations on extended molecules also. ${ }^{6}$

An interesting effect is observed in several of the examples studied, namely, the argon chain, the polyethylene and the isotactic polystyrene, where the negative of the HOMO energy (i.e., an uncorrelated and unrelaxed Koopmans theorem estimate of the ionization potential) is noticeably lower than the corresponding CIS excitation energy. This observation suggests that either the CIS/6-31G** treatment is inadequate or the physics of these system forbids bound excited states.

First we address the case of the polyethylene. The experimental values for the ionization potential and the band gap, which amount to 8.8 and $7.6 \mathrm{eVs}$, respectively, ${ }^{67}$ confirm the existence of bound states in this system. At the same time, the discrepancy between the experimental and calculated excitation energy, which is more than $4 \mathrm{eVs}$, is too large compared to the anticipated error of the CIS method of 1-2 eVs (see Refs. 68 or 69). This issue has already been investigated in Ref. 22. Calculations, as well as experiments on linear alkanes,${ }^{70}$ indicate that the lowest excited states in polyethylene are of a Rydberg type. This explains both the low values of the experimental or calculated ionization potential, and the inability of our calculation to reproduce the lowest excited states. In fact, a proper description of Rydberg states requires very diffuse basis functions, needed to represent the density of the excited electron perpendicular to the polymer chain. The authors of Ref. 22 did so by employing the 6-31++G basis and obtained a value of $9.2 \mathrm{eVs}$, which lies within the typical CIS error bar. The CIS states, calculated by employing the nondiffuse $6-31 \mathrm{G}^{* *}$ basis, which obviously allows for delocal- ization, but only along the polymer chain, are rather valence states and not related to the experimentally observed Rydberg states. Our code presently does not allow for using very diffuse basis functions for covalent polymers, because of convergence problems in the underlying Hartree-Fock calculations. In order to be able to study such Rydberg states we are working on an adaptation of the dual basis set scheme developed for LMP2 $2{ }^{60}$ However, in 3D closely packed systems Rydberg states are not possible and the need for diffuse basis functions vanishes. Low-lying excited states in 3D-crystals are expected to be of valence type.

For the case of the argon chain, owing to the sparser atomic arrangement, it was possible to include diffuse basis functions. We have added in the basis set an additional spshell with a relatively diffuse exponent of $0.07 \mathrm{Bohr}^{-2}$. The effect of this addition on the lowest excitation is dramatic: it reduces the value of the excitation energy from 21.7 to 12.9 eVs, well below the ionization potential, properly capturing the low-lying Rydberg states.

Finally, the effect of a lower ionization potential is also observed in the isotactic polystyrene, but not in the syndiotactic one. At the same time the excitation energy for the lowest state, which represents the $\pi \rightarrow \pi^{*}$ transition on the benzene rings, is virtually the same for both. A possible reason for the shift of the HOMO energy in the isotactic conformer is a noticeable elongation of the aliphatic $\mathrm{C}-\mathrm{C}$ bond (1.69 versus $1.55 \AA$ in the syndiotactic one) due to the mutual repulsion of the benzene rings.

As the timings in Table I show, the local CIS calculations for polymers are generally not very expensive and linear scaling allows one to increase the unit cell size without a big computational effort. The diffuseness of the excitons is a more demanding issue, but in the case of $1 \mathrm{D}$ systems it is still not a severe problem as far as the computational time is concerned. In 3D systems, however, the quadratic scaling can lead to a substantial loss in efficiency, since there it corresponds to a $R^{6}$ scaling with exciton localization radius $R$. As was mentioned above this undesired feature can be circumvented by the introduction of specific fit-domains for each product density to be fitted.

The direct space representation of an exciton provides an illustrative tool to analyze its locality. Figure 2 shows the histogram of the maximal CIS coefficients $\max _{i a}\left|c_{a \mathcal{A}}^{i}\right|$ for different cells $\mathcal{A}$. It is seen that the lowest energy exciton in $\mathrm{LiH}$ is well localized within a supercell consisting of 8-9 unit cells, which matches the 8-k-point convergence of the energy in Table I. The two conformers of polybiphenyl is another good example to analyze the exciton's locality. The singlet exciton in the parallel conformation, as seen in Table I, requires $14 \mathbf{k}$-points, while the perpendicular conformer only three (but with doubled unit cell). Again the direct space interpretation of this behavior can be observed in Fig. 2, where the exciton of the parallel polybiphenyl is localized within 15 unit cells, while its perpendicular counterpart is substantially more localized and "needs" just three doubled unit cells.

The local approach with nonorthogonal virtual states provides a very simple and useful indicator for the ability of the chosen k-mesh or the supercell to capture the studied exciton. As was shown in Sec. III B, the direct space norm of the 
CIS vector can be different from unity. This difference, however, tends to be zero when the values of the CIS vector at the edges of the supercell become small. Table II shows the convergence of the energies for the most diffuse exciton studied here with respect to the $\mathbf{k}$-mesh size, together with the values of the direct space norm.

Evidently, the energy and the norm smoothly converge with the number of $\mathbf{k}$-points. The norm is a good measure for the correctness of the description for a certain density of the $\mathbf{k}$-mesh. Furthermore, judging from the value of the norm at a given $\mathbf{k}$-point one can draw a conclusion about the diffuseness of the exciton under study. In Fig. 3 the dependence of the error in the direct space norm of singlet and triplet states for all the studied systems versus the number of the $\mathbf{k}$-points is displayed. In all cases the norm approaches unity more or less smoothly with the decay rate expressing the locality of the exciton.

One of the approximations of the current scheme lies in the evaluation of the two-electron integrals via the density fitting technique. While this approximation is essential for the efficiency of the method, the effect on the accuracy of the results should be negligible. The periodic density fitting scheme

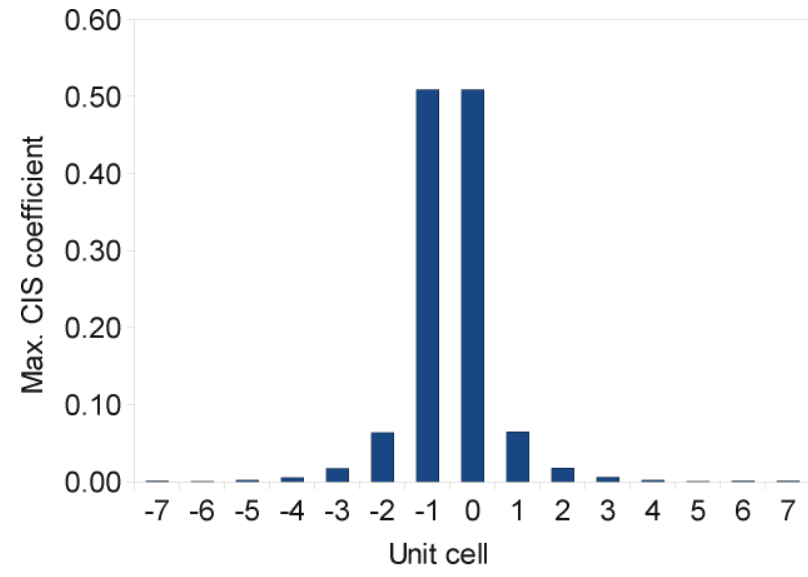

(i) $\mathrm{LiH}$

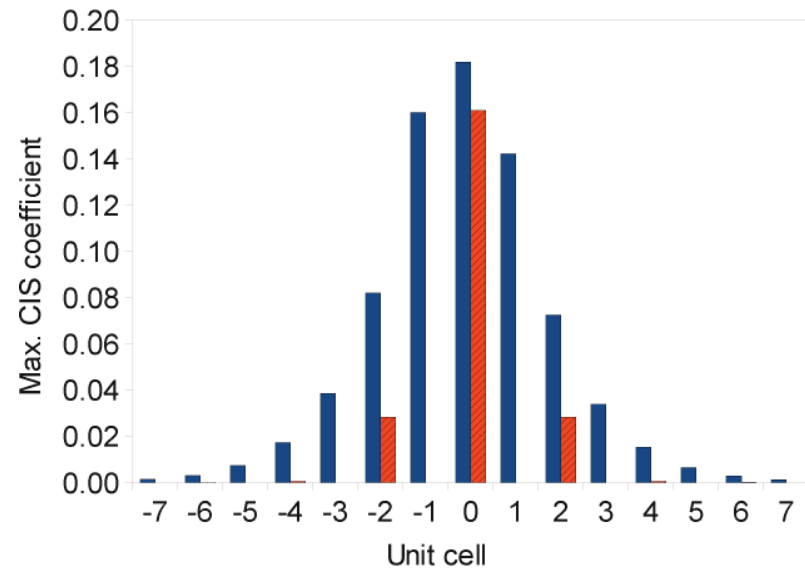

(ii) Biphenyl

FIG. 2. Maximal CIS coefficients per cell (singlet state) for (i) $\mathrm{LiH}$ and (ii) biphenyl (solid: parallel; dashed: perpendicular).
TABLE II. Excitation energies and the direct-space norm of the CIS vector for polybiphenyl (parallel) calculated with 6-31G orbital basis set (with upscaled exponent of the p-function of carbon) and VDZ fitting basis set.

\begin{tabular}{lllllr}
\hline \hline & \multicolumn{2}{c}{ Excitation energy / eV } & & \multicolumn{2}{c}{ Norm of the CIS vector } \\
\cline { 2 - 3 } \cline { 6 - 6 }$n_{\mathbf{k}}$ & Singlet & Triplet & & Singlet & Triplet \\
\hline 5 & 3.7133 & 2.1438 & & 0.98055 & 0.99319 \\
6 & 3.7783 & 2.1745 & & 0.98736 & 0.99562 \\
7 & 3.8029 & 2.1828 & & 0.99481 & 0.99869 \\
8 & 3.8205 & 2.1886 & & 0.99697 & 0.99923 \\
9 & 3.8273 & 2.1904 & & 0.99884 & 0.99978 \\
10 & 3.8315 & 2.1914 & & 0.99936 & 0.99987 \\
11 & 3.8331 & 2.1918 & & 0.99977 & 0.99997 \\
12 & 3.8340 & 2.1919 & & 0.99988 & 0.99998 \\
13 & 3.8344 & 2.1920 & 0.99996 & 1.00000 \\
14 & 3.8345 & 2.1920 & 0.99998 & 1.00000 \\
15 & 3.8346 & 2.1921 & 0.99999 & 1.00000 \\
16 & 3.8347 & 2.1921 & 1.00000 & 1.00000 \\
\hline \hline
\end{tabular}

of the ground state correlation calculations have been shown to cause errors in the energy of about $10^{-5}$ Hartree per atom, ${ }^{43}$ provided that the quality of the fitting basis set matches that of the orbital basis set. In the present study we used orbital basis sets of the double-zeta quality. Table III, which lists the energies, obtained by employing fitting basis sets of increasing quality, shows that the density fitting errors are indeed tiny.
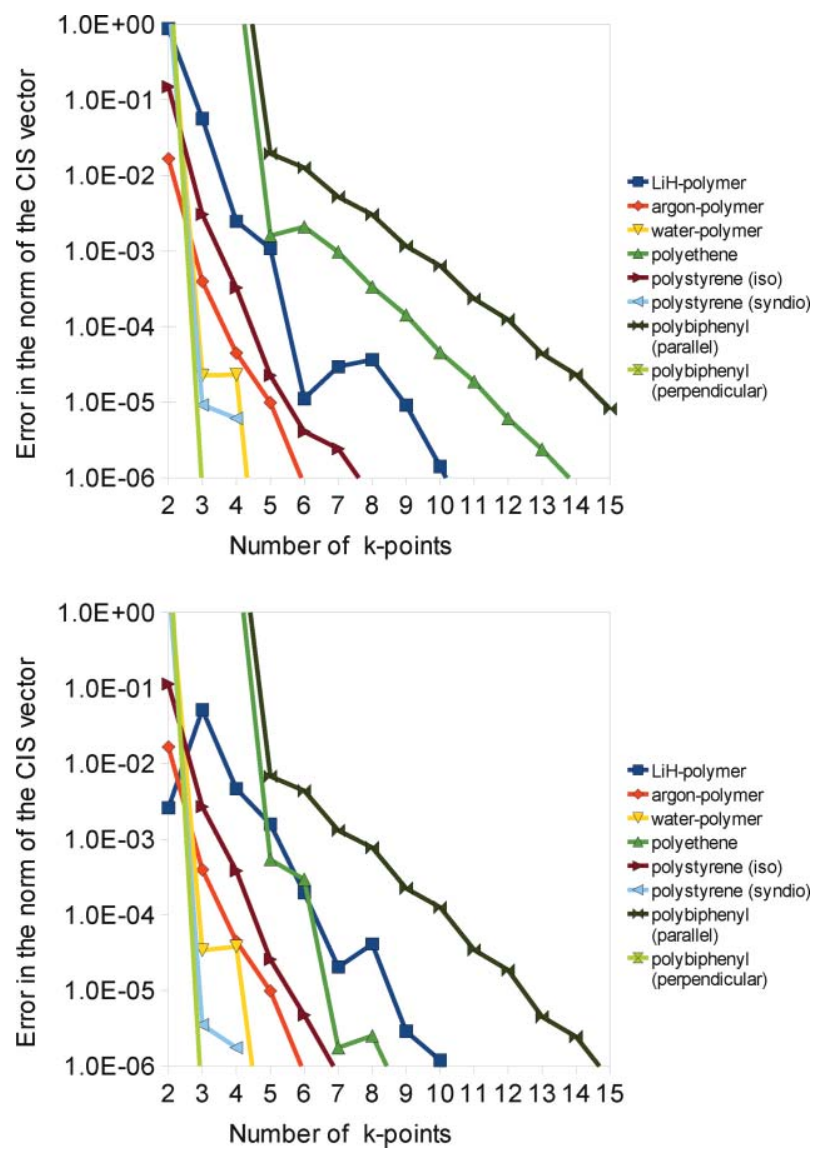

FIG. 3. Errors in the direct space norm for singlet (top) and triplet (bottom) exciton states of different systems. 
TABLE III. Singlet-Triplet CIS excitation energies with the $6-31 \mathrm{G}^{* *}$ AO basis and fitting basis sets of different quality. GTO-fitting basis sets, optimized for MP2 calculations with cc-pVXZ orbitals basis sets and then converted to mixed PTO/GTO as explained in Ref. 43, are used and denoted as VXZ.

\begin{tabular}{lrrrrrrr}
\hline \hline & \multicolumn{3}{c}{ Singlet/eV } & & \multicolumn{3}{c}{ Triplet / eV } \\
\cline { 2 - 4 } \cline { 7 - 8 } System & \multicolumn{1}{c}{ VDZ } & \multicolumn{1}{c}{ VTZ } & \multicolumn{1}{c}{ VQZ } & & VDZ & \multicolumn{1}{c}{ VTZ } & \multicolumn{1}{c}{ VQZ } \\
\hline $\mathrm{LiH}$ & 6.6060 & 6.6058 & 6.6060 & & 5.5021 & 5.5018 & 5.5019 \\
$\mathrm{Ar}$ & 21.7344 & 21.7337 & 21.7339 & & 20.5186 & 20.5177 & 20.5178 \\
$\mathrm{H}_{2} \mathrm{O}$ & 10.3781 & 10.3792 & 10.3793 & & 9.4848 & 9.4849 & 9.4848 \\
$\mathrm{C}_{2} \mathrm{H}_{4}$ & 11.7476 & 11.7477 & 11.7477 & & 10.5475 & 10.5475 & 10.5475 \\
\hline \hline
\end{tabular}

It is worth mentioning that despite the presence of the two-external three-index integrals, which are absent in the MP2 method, the auxiliary basis sets optimized for MP2 seem to be perfectly applicable for CIS. We cannot compare our results directly to those obtained without the density fitting approximation, since the conventional four-index integral generator has not been implemented. However, our method exactly reproduces the results of the polyethylene as reported in Ref. 26, which were obtained with the canonical nondensity-fitted CIS method (see the supplementary material $\left.{ }^{66}\right)$.

Finally, we focus our attention on the influence of the starting CIS vector on the number of the Davidson iterations. As discussed in Sec. III E the direct-space guesses have the advantage of the possibility to enforce the locality on the starting vector, but they suffer from having no (guess 1 and 2) or little (guess 3 ) information on the actual nature of the exciton. The reciprocal starting guess 4 on the contrary includes some knowledge of the excitonic behavior, but can be delocalized artificially. As is seen from Table IV, for the examples presented here the reciprocal space guess, in fact, turns out to be the most efficient. Guess 3, although including several starting vectors, rather than one, in most cases does not lead to any improvement even in comparison to the guesses 1 or 2 .

\section{CONCLUSIONS}

The periodic local density fitted CIS method presented in this contribution is the first step toward correlated optical band gaps described in the framework of local correlation methods. It explicitly includes the excitonic phenomenon, i.e., the

TABLE IV. The number of iterations needed to calculate the lowest singlet exciton state using VDZ fitting basis set.

\begin{tabular}{lcccc}
\hline \hline & \multicolumn{4}{c}{ Number of iterations for guess } \\
\cline { 2 - 5 } System & 1 & 2 & 3 & 4 \\
\hline $\mathrm{LiH}$ & 15 & 15 & $5+7$ & 11 \\
$\mathrm{Ar}$ & 15 & 15 & $5+7$ & 10 \\
$\mathrm{H}_{2} \mathrm{O}$ & 30 & 20 & $5+23$ & 8 \\
$\mathrm{C}_{2} \mathrm{H}_{4}$ & 31 & 32 & $5+31$ & 11 \\
Styrene (iso) & 21 & 18 & $5+27$ & 14 \\
Styrene (syn) & 37 & 38 & $5+35$ & 12 \\
Biphenyl (II) & 20 & 16 & $5+23$ & 9 \\
Biphenyl $(\perp)$ & 36 & 32 & $5+27$ & 17 \\
\hline \hline
\end{tabular}

attraction between the hole and the electron. The screening effects due to electron correlation are, however, absent in this approach, which might lead to errors in the excitation energies of more than an electron volt. A correlation treatment, which corrects this deficiency, is planned to be added on top of the CIS along the lines of local correlation schemes. Indeed, for molecular excited states dominated by single excitations, the CIS method is known to usually provide a good description of the nature of these states, and is thus used as a starting point in correlated excited state treatments (like in TD-CC response theory). Furthermore, the ability of the periodic CIS method alone to provide a semiquantitative theoretical description of the excitonic states makes this method useful in its own right, also, due to a relative scarcity of inexpensive practical tools to study such states in the solid state computational science. Presently, the method is capable of treating $\Gamma$-point excitons in polymers with up to several dozens of atoms in the cell. Work on generalizing this approach to 2D- and 3Dperiodic systems, and for any $\mathbf{k}$-point of the Brillouin zone is underway.

\section{ACKNOWLEDGMENTS}

The authors thank Dr. Kats, Dr. Maschio, and Professor Pisani for useful discussions. Financial support from the Deutsche Forschungsgemeinschaft (DFG), Schwerpunktprogramm 1145 , is gratefully acknowledged.

${ }^{1}$ O. Christiansen, P. Jørgensen, and C. Hättig, Int. J. Quantum Chem. 68, 1 (1998).

${ }^{2}$ A. Trofimov and J. Schirmer, J. Phys. B 28, 2299 (1995).

${ }^{3}$ M. Head-Gordon, R. J. Rico, M. Oumi, and T. J. Lee, Chem. Phys. Lett. 219, 21 (1994).

${ }^{4}$ B. O. Roos, M. P. Fülscher, P.-A. Malmqvist, M. Merchán, and L. SerranoAndrés, in Quantum Mechanical Electronic Structure Calculations with Chemical Acuracy, edited by S. R. Langhoff (Kluwer Academic Publishers, Dordrecht, The Netherlands, 1995), p. 357.

${ }^{5}$ D. Kats, T. Korona, and M. Schütz, J. Chem. Phys. 125, 104106 (2006).

${ }^{6}$ K. Freundorfer, D. Kats, T. Korona, and M. Schütz, J. Chem. Phys. 133, $244110(2010)$

${ }^{7}$ P. Y. Ayala, K. N. Kudin, and G. E. Scuseria, J. Chem. Phys. 115, 9698 (2001).

${ }^{8}$ C. Pisani, L. Maschio, S. Casassa, M. Halo, M. Schütz, and D. Usvyat, J. Comp. Chem. 29, 2113 (2008).

${ }^{9}$ M. Marsman, A. Gruneis, J. Paier, and G. Kresse, J. Chem. Phys. 130, 184103 (2009).,

${ }^{10}$ H. Stoll, Phys. Rev. B 46, 6700 (1992).

${ }^{11}$ B. Paulus, Phys. Rep. 428, 1 (2006).

${ }^{12}$ S. Nolan, M. Gillan, D. Alfe, N. Allan, and F. Manby, Phys. Rev. B 80, 165109 (2009).

${ }^{13}$ A. Stoyanova, L. Hozoi, P. Fulde, and H. Stoll, J. Chem. Phys. 131, 044119 (2009).

${ }^{14}$ S. Suhai, Phys. Rev. B 29, 4570 (1984).

${ }^{15}$ S. Suhai, Int. J. Quantum Chem. 29, 469 (1986).

${ }^{16}$ G. Onida, L. Reining, and A. Rubio, Rev. Mod. Phys. 74, 601 (2002).

${ }^{17}$ M. Rohlfing and S. G. Louie, Phys. Rev. Lett. 81, 2312 (1998).

${ }^{18}$ M. Rohlfing and S. G. Louie, Phys. Rev. Lett. 82, 1959 (1999).

${ }^{19}$ M. J. Lucero, A. M. N. Niklasson, S. Tretiak, and M. Challacombe, J. Chem. Phys. 129, 064114 (2008).

${ }^{20} \mathrm{C}$. Friedrich and A. Schindlmayr, in Computational Nanoscience: Do It Yourself!, edited by J. Grotendorst, S. Blugel, and D. Marx (John von Neumann Institute for Computing, Julich, Germany, 2006), pp. 335-355.

${ }^{21}$ S. Suhai, Int. J. Quantum Chem. 26, 223 (1984).

${ }^{22}$ S. Hirata and R. Bartlett, J. Chem. Phys. 112, 7339 (2000). 
${ }^{23}$ S. Hirata and T. Shimazaki, Phys. Rev. B. 80, 085118 (2009).

${ }^{24}$ A. Gruneis, M. Marsman, and G. Kresse, J. Chem. Phys. 133, 074107 (2010).

${ }^{25}$ M. G. Vracko and M. Zaider, Int. J. Quantum Chem. 43, 321 (1992).

${ }^{26}$ S. Hirata, M. Head-Gordon, and R. J. Bartlett, J. Chem. Phys. 111, 10774 (1999).

${ }^{27}$ C. Ko, D. K. Malick, D. A. Braden, R. A. Friesner, and T. J. Martínez, J. Chem. Phys. 128, 104103 (2008).

${ }^{28}$ M. G. Vracko and M. Zaider, Int. J. Quantum Chem. 47, 119 (1993).,

${ }^{29}$ M. Vracko, B. Champagne, D. H. Mosley, and J.-M. André, J. Chem. Phys. 102, 6831 (1995).

${ }^{30}$ H. Katagiri, J. Chem. Phys. 122, 224901 (2005).

${ }^{31}$ W. Hanke and L. J. Sham, Phys. Rev. B. 12, 4501 (1975).

${ }^{32}$ P. H. Hahn, W. G. Schmidt, K. Seino, M. Preuss, F. Bechsted, and J. Bernholc, Phys. Rev. Lett. 94, 037404 (2005).

${ }^{33}$ M. Shishkin, M. Marsman, and G. Kresse, Phys. Rev. Lett. 99, 246403 (2007).

${ }^{34}$ K. Hummer, P. Puschnig, and C. Ambrosch-Draxl, Phys. Rev. Lett. 92, 147402 (2004).

${ }^{35}$ F. Sottile, M. Marsili, V. Olevano, and L. Reining, Phys. Rev. B 76, 161103 (2007).

${ }^{36}$ M. Palummo, O. Pulci, R. D. Sole, A. Marini, P. Hahn, W. Schmidt, and F. Bechstedt, J. Phys.: Condens. Matter 16, S4313 (2004).

${ }^{37}$ A. Izmaylov and G. Scuseria, J. Chem. Phys. 129, 034101 (2008).

${ }^{38}$ G. H. Wannier, Phys. Rev. 52, 191 (1937).

${ }^{39}$ S. Saebø and P. Pulay, Chem. Phys. Lett. 113, 13 (1985).

${ }^{40}$ M. Schütz, G. Hetzer, and H. J. Werner, J. Chem. Phys. 111, 5691 (1999).

${ }^{41}$ L. Maschio, D. Usvyat, F. Manby, S. Casassa, C. Pisani, and M. Schütz, Phys. Rev. B 76, 075101 (2007).

${ }^{42}$ L. Maschio and D. Usvyat, Phys. Rev. B 78, 073102 (2008).

${ }^{43}$ M. Schütz, D. Usvyat, M. Lorenz, C. Pisani, L. Maschio, S. Casassa, and M. Halo, in Accurate Condensed Phase Quantum Chemistry, edited by F. R. Manby (CRC Press, NY, 2010), p. 27.

${ }^{44}$ E. R. Davidson, J. Comput. Phys. 17, 87 (1975).

${ }^{45}$ B. O. Roos and P. E. M. Siegbahn, in Modern Theoretical Chemistry, Methods of Electronic Structure, edited by H. F. Schaefer (Plenum Press, New York, 1977), Vol. 3, pp. 277-318.

${ }^{46}$ C. M. Zicovich-Wilson, R. Dovesi, and V. R. Saunders, J. Chem. Phys. 115, 9708 (2001)

${ }^{47}$ N. Marzari and D. Vanderbilt, Phys. Rev. B 56, 12847 (1997).
${ }^{48}$ R. Dovesi, V. R. Saunders, R. Roetti, R. Orlando, C. M. Zicovich-Wilson, F. Pascale, B. Civalleri, K. Doll, N. M. Harrison, I. J. Bush, P. D'Arco, and M. Llunell, CRYSTAL09 User's Manual (Università di Torino, Torino, 2009).

${ }^{49}$ J. D. Cloizeaux, Phys. Rev. 135, A685 (1964).

${ }^{50}$ D. Kats, D. Usvyat, and M. Schütz, Phys. Chem. Chem. Phys. 10, 3430 (2008).

${ }^{51}$ B. I. Dunlap, Phys. Chem. Chem. Phys. 2, 2113 (2000).

${ }^{52}$ R. Evarestov and I. Tupitsyn, Phys. Solid State 44, 1656 (2002).

${ }^{53}$ S. B. Trickey, J. A. Alford, and J. C. Boettger, Computational Materials Science, Theoretical and Computational Chemistry series, edited by J. Leszczynski (Elsevier, Oxford, 2004), Vol. 15, p. 171.

${ }^{54}$ S. Varga, M. Milko, and J. Noga, J. Chem. Phys. 124, 034106 (2006).

${ }^{55}$ A. Burow, M. Sierka, and F. Mohamed, J. Chem. Phys. 131, 214101 (2009).

${ }^{56}$ H. J. Werner, F. R. Manby, and P. J. Knowles, J. Chem. Phys. 118, 8149 (2003).

${ }^{57}$ A. Izmaylov and G. Scuseria, Phys. Chem. Chem. Phys. 10, 3421 (2008).

${ }^{58}$ M. Schütz and F. R. Manby, Phys. Chem. Chem. Phys. 5, 3349 (2003).

${ }^{59}$ S. Casassa, C. M. Zicovich-Wilson, and C. Pisani, Theor. Chem. Acc. 116, 726 (2006).

${ }^{60}$ D. Usvyat, L. Maschio, C. Pisani, and M. Schütz, Z. Phys. Chem. 224, 441 (2010).

${ }^{61}$ F. R. Manby, P. J. Knowles, and A. W. Lloyd, J. Chem. Phys. 115, 9144 (2001).

${ }^{62}$ J. W. Mintmire and B. I. Dunlap, Phys. Rev. A 25, 88 (1982).

${ }^{63}$ F. R. Manby and P. J. Knowles, Phys. Rev. Lett. 87, 163001 (2001).

${ }^{64}$ W. Kohn, Phys. Rev. 115, 809 (1959).

${ }^{65}$ J. D. Cloizeaux, Phys. Rev. 135, A698 (1964).

${ }^{66}$ See supplementary material at http://dx.doi.org/10.1063/1.3554209 for the structural parameters of the studies systems.

${ }^{67}$ K. J. Less and E. G. Wilson, J. Phys. C 6, 3110 (1973).

${ }^{68}$ O. Christiansen, H. Koch, and P. Jørgensen, Chem. Phys. Lett. 243, 409 (1995).

${ }^{69}$ C. Hättig, Advances in Quantum Chemistry (Elsevier, Oxford, 2005) Vol. 50, p. 37.

${ }^{70}$ E. A. Costner, B. K. Long, C. Navar, S. Jockusch, X. Lei, P. Zimmerman, A. Campion, N. J. Turro, and C. G. Willson, J. Phys. Chem. A 113, 9337 (2009). 